\title{
Up-down symmetry of the turbulent transport of toroidal angular momentum in tokamaks
}

\author{
Felix I. Parra, ${ }^{1,2}$ Michael Barnes, ${ }^{1,3,2}$ and Arthur G. Peeters ${ }^{4,2}$ \\ ${ }^{1}$ Rudolf Peierls Centre for Theoretical Physics, University of Oxford, Oxford, OX1 3NP, UK \\ ${ }^{2}$ Isaac Newton Institute for Mathematical Sciences, Cambridge CB3 OEH, UK \\ ${ }^{3}$ Euratom/CCFE Fusion Association, Culham Science Centre, Abingdon OX14 3DB, UK \\ ${ }^{4}$ Universität Bayreuth, 95440 Bayreuth, Germany
}

(Dated: June 2, 2021)

\begin{abstract}
Two symmetries of the local nonlinear $\delta f$ gyrokinetic system of equations in tokamaks in the high flow regime are presented. The turbulent transport of toroidal angular momentum changes sign under an up-down reflection of the tokamak and a sign change of both the rotation and the rotation shear. Thus, the turbulent transport of toroidal angular momentum must vanish for updown symmetric tokamaks in the absence of both rotation and rotation shear. This has important implications for the modeling of spontaneous rotation.
\end{abstract}

PACS numbers: $52.30 . \mathrm{Gz}, 52.65 . \mathrm{Tt}$

\section{INTRODUCTION}

Understanding how momentum is distributed within a tokamak is crucial to explaining experimentally observed regions of reduced turbulent transport like Internal Transport Barriers (ITBs) [1] and edge pedestals [2]. In these regions of reduced transport, turbulence is suppressed by strong velocity gradients, which are determined by the transport of toroidal angular momentum. Nonlinear $\delta f$ flux tube simulations [3 6] are extensively used to study turbulent transport of toroidal angular momentum in tokamaks [7-14. The time averaged turbulent transport of momentum is found to be zero for updown symmetric tokamaks in the absence of toroidal velocity and toroidal velocity gradient. This behavior was addressed in Ref. [15], where the transport of parallel momentum due to electrostatic fluctuations was studied with a quasilinear model. Ref. [15] showed that the linearized gyrokinetic equation has a symmetry that results in the transport of parallel momentum vanishing for updown symmetric tokamaks in the absence of velocity and velocity shear. Moreover, this analysis was successfully employed to identify the up-down asymmetry as a drive for momentum transport [9].

However, there are six major effects that were not treated in the analysis of Ref. [15. First, the symmetry given in Ref. [15] is only valid for the linearized equation. Second, the formulation was collisionless. Third, the effect of the $\mathbf{E} \times \mathbf{B}$ shear on transport of momentum [10, 11, 16] was not considered. Fourth, the Mach number was assumed to be small and the effect of the centrifugal force [12] was neglected. Fifth, in the evaluation of the radial transport of toroidal angular momentum, the contribution of the component of the velocity perpendicular to the magnetic field was neglected. Sixth, electromagnetic effects were not taken into account. In this article, we present the complete symmetry argument. We consider the high flow regime, for which the mean velocity is comparable to the thermal speed of the ions, and we use the local approximation, for which the turbulent transport only depends on local quantities. In this limit, the sign of the radial transport of toroidal angular momentum changes under a sign change of the average velocity and its gradient and an up-down reflection. Thus, for an up-down symmetric tokamak without any velocity or velocity shear, the momentum transport is zero.

The symmetry presented here is broken if higher order terms in the gyrokinetic expansion in $\epsilon_{i}=\rho_{i} / a \ll 1$ are kept, giving transport of momentum that is small in $\epsilon_{i}$ compared to the high flow results. Here $\rho_{i}$ is the ion gyroradius and $a$ is the minor radius of the tokamak. The effect of these higher order terms on transport of momentum is discussed in Ref. [17, where a higher order equation valid in the limit of small poloidal magnetic field is proposed. The complete higher order gyrokinetic Fokker-Planck equation valid for any magnetic geometry was self-consistently calculated for the first time in Ref. [18] for the electrostatic approximation.

The remainder of this article is organized as follows. We first present the local $\delta f$ gyrokinetic model in the high flow regime in section [II. We then show in section III] that the gyrokinetic equations have two fundamental symmetries that involve an up-down reflection. Finally, the consequences of these symmetries for transport of momentum are discussed in section [V] The most cumbersome parts of the calculation are given in Appendices $\mathrm{A}, \mathrm{G}$.

\section{LOCAL $\delta f$ GYROKINETIC FORMULATION}

In this section we present the local $\delta f$ gyrokinetic system of equations in the high flow ordering, for which the average velocity of the ions $\mathbf{V}_{i}$ is of the order of the ion thermal speed $v_{t i}$.

First, in subsection [IA we review the magnetohydrodynamic (MHD) equilibrium for a tokamak and we discuss the background density, temperature and velocity. We then present in subsection IIB the system of equa- 
tions for the fluctuating pieces of the distribution function and the electromagnetic field. These equations are simplified in the local limit in subsection II C. We finish by giving the radial flux of toroidal angular momentum as a function of the fluctuating distribution function and electromagnetic fields in subsection IID.

\section{A. Equilibrium}

The background axisymmetric magnetic field of a tokamak is given by

$$
\mathbf{B}=I \nabla \zeta+\nabla \zeta \times \nabla \psi
$$

where $\psi$ is the poloidal magnetic flux, $\zeta$ is the toroidal angle, $\nabla \zeta=\hat{\zeta} / R$, with $\hat{\boldsymbol{\zeta}}$ the unit vector in the toroidal direction and $R$ the major radius, and $I=R B_{\zeta}$, with $B_{\zeta}$ the toroidal magnetic field. We use $\psi, \zeta$ and a poloidal angle $\theta$ as our coordinates. The Jacobian of the transformation from cartesian coordinates $\mathbf{x}$ into $\psi, \theta$ and $\zeta$ is

$$
\mathcal{J}=|(\nabla \psi \times \nabla \theta) \cdot \nabla \zeta|^{-1}=|\mathbf{B} \cdot \nabla \theta|^{-1}
$$

where we take the absolute value to avoid having a sign in the Jacobian.

In the high flow ordering, the electric field is electrostatic and purely radial to lowest order, and the potential is given by $e \Phi_{-1}(\psi, t) / T_{e} \sim \epsilon_{i}^{-1}$, with $T_{e}$ the electron temperature and $e$ the magnitude of the electron charge. To lowest order, all species rotate at the same speed within a flux surface, i.e., $\mathbf{V}_{s}=\mathbf{V}_{i}=R \Omega_{\zeta}(\psi, t) \hat{\boldsymbol{\zeta}} \sim v_{t i}$ [19 21], where the subscript $s$ indicates the species, and $\Omega_{\zeta}(\psi, t)=-c\left(\partial \Phi_{-1} / \partial \psi\right)$ is the rotation frequency. To next order, there is an axisymmetric component of the potential, $e \Phi_{0}(\psi, \theta, t) / T_{e} \sim 1$ that ensures quasineutrality within a flux surface.

It is convenient to solve the equations in the frame rotating with $\Omega_{\zeta}$. Thus, from now on we use the peculiar velocity $\mathbf{w}=\mathbf{v}-R \Omega_{\zeta} \hat{\boldsymbol{\zeta}}$, where $\mathbf{v}$ is the velocity in the laboratory frame. In the frame rotating with $\Omega_{\zeta}$, the lowest order distribution functions are stationary Maxwellians $f_{M s}=n_{s}\left(m_{s} / 2 \pi T_{s}\right)^{3 / 2} \exp \left(-m_{s} w^{2} / 2 T_{s}\right)$, where $m_{s}$ is the species mass, and $n_{s}$ and $T_{s}$ are the species density and temperature, respectively. The temperatures $T_{s}(\psi, t)$ are flux functions. Due to the centrifugal force and the electrostatic potential, the densities are not constant in a flux surface. Instead, they are given by

$$
n_{s}(\psi, \theta, t)=\eta_{s}(\psi, t) \exp \left(-\frac{Z_{s} e \Phi_{0}}{T_{s}}+\frac{m_{s} \Omega_{\zeta}^{2} R^{2}}{2 T_{s}}\right) \text {, }
$$

with $\eta_{s}(\psi, t)$ a flux function and $Z_{s} e$ the species charge. Imposing quasineutrality,

$$
\sum_{s} Z_{s} e n_{s}=0
$$

gives the piece of the electrostatic potential $\Phi_{0}(\psi, \theta, t)$.

With these lowest order equilibrium distribution functions, $I(\psi, t)=R B_{\zeta}$ is a flux function, and the poloidal component of the magnetic field is determined by the Grad-Shafranov equation

$$
R^{2} \nabla \cdot\left(\frac{\nabla \psi}{R^{2}}\right)=-I \frac{\partial I}{\partial \psi}-\left.4 \pi R^{2} \frac{\partial p}{\partial \psi}\right|_{R}
$$

where the pressure is

$$
p(\psi, \theta, t)=\sum_{s} \eta_{s} T_{s} \exp \left(-\frac{Z_{s} e \Phi_{0}}{T_{s}}+\frac{m_{s} \Omega_{\zeta}^{2} R^{2}}{2 T_{s}}\right)
$$

and

$$
\left.\frac{\partial p}{\partial \psi}\right|_{R}=\frac{\partial p}{\partial \psi}-\sum_{s} n_{s} m_{s} \Omega_{\zeta}^{2} R \frac{\partial R}{\partial \psi}
$$

\section{B. Equations for the turbulent fluctuations}

The $\delta f$ formulation in the high flow regime is discussed in 22 25]. It determines the turbulent pieces of the distribution function, $\delta \bar{f}_{s}(\mathbf{x}, \mathbf{w}, t) \sim \epsilon_{i} f_{M s}$, the electrostatic potential, $\bar{\phi}(\mathbf{x}, t) \sim \epsilon_{i} T_{e} / e$, the parallel component of the vector potential, $\bar{A}_{\|}(\mathbf{x}, t) \sim \epsilon_{i} \rho_{i} B$, and the parallel component of the magnetic field, $\bar{B}_{\|}(\mathbf{x}, t) \sim \epsilon_{i} B$. The perturbed electric and magnetic fields in the frame rotating with velocity $\Omega_{\zeta}$ are to lowest order $\delta \overline{\mathbf{E}}=-\nabla_{\perp} \bar{\phi}$ and $\delta \overline{\mathbf{B}}=\nabla_{\perp} \bar{A}_{\|} \times \hat{\mathbf{b}}+\bar{B}_{\| \mid} \hat{\mathbf{b}}$, where $\hat{\mathbf{b}}=\mathbf{B} / B$ is the unit vector in the direction of the background magnetic field, and $\nabla_{\perp}=\nabla-\hat{\mathbf{b}} \hat{\mathbf{b}} \cdot \nabla$ is the gradient perpendicular to the background magnetic field. We assume that the perpendicular gradient of the fluctuations is large, on the order of the inverse of the ion gyroradius, $\nabla_{\perp} \sim 1 / \rho_{i}$, whereas the parallel gradient is comparable to the inverse of the characteristic length of the device, $\hat{\mathbf{b}} \cdot \nabla \sim 1 / a$. The overline ${ }^{-}$over the perturbed quantities will be useful later because the quantities without this overline will be their Fourier transforms.

To eliminate the fast gyrofrequency time scale, it is necessary to change to gyrokinetic phase space coordinates. We use the guiding center position $\mathbf{X}=\mathbf{x}+$ $\Omega_{s}^{-1} \mathbf{w} \times \hat{\mathbf{b}}$, the velocity parallel to the magnetic field $w_{\|}=\mathbf{w} \cdot \hat{\mathbf{b}}$, the magnetic moment $\mu=w_{\perp}^{2} / 2 B$ and the gyrophase $\varphi=\arctan [(\mathbf{w} \times \hat{\mathbf{b}}) \cdot \nabla \psi /(\mathbf{w} \cdot \nabla \psi)]$, where $\Omega_{s}=Z_{s} e B / m_{s} c$ is the species gyrofrequency and $\mathbf{w}_{\perp}=$ $\mathbf{w}-w_{\|} \hat{\mathbf{b}}$ is the velocity perpendicular to the background magnetic field. With these new coordinates, it is easy to average out the gyrofrequency time scale. Extracting the Maxwell-Boltzmann response from the distribution function, i.e., $\delta \bar{f}_{s}=\bar{h}_{s}-\left(Z_{s} e \bar{\phi} / T_{s}\right) f_{M s}, \bar{h}_{s}\left(\mathbf{X}, w_{\|}, \mu, t\right)$ can be proven to be gyrophase independent, and the $\delta f$ kinetic equation becomes

$$
\frac{\partial \bar{h}_{s}}{\partial t}+R \Omega_{\zeta} \hat{\boldsymbol{\zeta}} \cdot \nabla_{\mathbf{X}} \bar{h}_{s}+w_{\|} \hat{\mathbf{b}} \cdot \nabla_{\mathbf{X}} \bar{h}_{s}+\mathbf{v}_{d, s} \cdot \nabla_{\mathbf{X}} \bar{h}_{s}
$$




$$
\begin{array}{r}
+a_{\|, s} \frac{\partial \bar{h}_{s}}{\partial w_{\|}}-\sum_{s^{\prime}}\left\langle\bar{C}_{s s^{\prime}}^{(\ell)}\right\rangle_{s}+\overline{\mathbf{v}}_{\chi, s} \cdot \nabla_{\mathbf{X}} \bar{h}_{s} \\
=\frac{Z_{s} e f_{M s}}{T_{s}}\left(\frac{\partial\langle\bar{\chi}\rangle_{s}}{\partial t}+R \Omega_{\zeta} \hat{\boldsymbol{\zeta}} \cdot \nabla_{\mathbf{X}}\langle\bar{\chi}\rangle_{s}\right) \\
-\overline{\mathbf{v}}_{\chi, s} \cdot\left[\nabla_{\mathbf{X}} f_{M s}+\frac{m_{s} f_{M s}}{T_{s}}\left(\frac{I w_{\|}}{B} \nabla_{\mathbf{X}} \Omega_{\zeta}\right.\right. \\
\left.\left.+\frac{Z_{s} e}{m_{s}} \nabla_{\mathbf{X}} \Phi_{0}-R \Omega_{\zeta}^{2} \nabla_{\mathbf{X}} R\right)\right]
\end{array}
$$

where the Maxwellian is written in the new variables as $f_{M s}=n_{s}\left(m_{s} / 2 \pi T_{s}\right)^{3 / 2} \exp \left[-m_{s}\left(w_{\|}^{2} / 2+\mu B\right) / T_{s}\right]$. The drift

$$
\overline{\mathbf{v}}_{\chi, s}=-\frac{c}{B} \nabla_{\mathbf{X}}\langle\bar{\chi}\rangle_{s} \times \hat{\mathbf{b}}
$$

accounts for the parallel motion along the perturbed magnetic field lines and for the turbulent $\mathbf{E} \times \mathbf{B}$ and $\nabla B$ drifts. The generalized potential $\langle\bar{\chi}\rangle_{s}$ is given by

$$
\begin{array}{r}
\langle\bar{\chi}\rangle_{s}=\left\langle\bar{\phi}\left(\mathbf{X}+\boldsymbol{\rho}_{s}, t\right)\right\rangle_{s}-\frac{1}{c} w_{\|}\left\langle\bar{A}_{\|}\left(\mathbf{X}+\boldsymbol{\rho}_{s}, t\right)\right\rangle_{s} \\
-\frac{1}{c}\left\langle\mathbf{w}_{\perp} \cdot \overline{\mathbf{A}}_{\perp}\left(\mathbf{X}+\boldsymbol{\rho}_{s}, t\right)\right\rangle_{s},
\end{array}
$$

where $\overline{\mathbf{A}}_{\perp}$ is related to $\bar{B}_{\|}$by $\bar{B}_{\|}=\hat{\mathbf{b}} \cdot\left(\nabla \times \overline{\mathbf{A}}_{\perp}\right)$ and $\langle\ldots\rangle_{s}$ is the gyroaverage holding $\mathbf{X}, w_{\|}, \mu$ and $t$ fixed. The dependence of $\bar{\phi}, \bar{A}_{\|}$and $\overline{\mathbf{A}}_{\perp}$ on the gyrophase is through $\mathbf{x}=\mathbf{X}+\boldsymbol{\rho}_{s}$ that contains the gyroradius $\boldsymbol{\rho}_{s}=$ $-\Omega_{s}^{-1} \mathbf{w} \times \hat{\mathbf{b}}$, also given by

$$
\boldsymbol{\rho}_{s}(\mu, \varphi)=\frac{\sqrt{2 \mu B}}{\Omega_{s}|\nabla \psi|}[-\sin \varphi \nabla \psi+\cos \varphi(\hat{\mathbf{b}} \times \nabla \psi)] .
$$

The perpendicular drifts $\mathbf{v}_{d, s}$ are

$$
\mathbf{v}_{d, s}=\mathbf{v}_{E 0}+\mathbf{v}_{M, s}+\mathbf{v}_{c o, s}+\mathbf{v}_{c f, s}
$$

where $\mathbf{v}_{E 0}=-(c / B) \nabla_{\mathbf{X}} \Phi_{0} \times \hat{\mathbf{b}}$ is the $\mathbf{E} \times \mathbf{B}$ drift due to the background axisymmetric potential $\Phi_{0}, \mathbf{v}_{M, s}=$ $\left(\mu / \Omega_{s}\right) \hat{\mathbf{b}} \times \nabla_{\mathbf{X}} B+\left(w_{\|}^{2} / \Omega_{s}\right) \hat{\mathbf{b}} \times\left(\hat{\mathbf{b}} \cdot \nabla_{\mathbf{X}} \hat{\mathbf{b}}\right)$ are the $\nabla B$ and curvature drifts, $\mathbf{v}_{c o, s}=\left(2 w_{\|} \Omega_{\zeta} / \Omega_{s}\right) \hat{\mathbf{b}} \times\left[\left(\nabla_{\mathbf{X}} R \times \hat{\boldsymbol{\zeta}}\right) \times \hat{\mathbf{b}}\right]$ is the Coriolis drift, and $\mathbf{v}_{c f, s}=-\left(R \Omega_{\zeta}^{2} / \Omega_{s}\right) \hat{\mathbf{b}} \times \nabla_{\mathbf{X}} R$ is the centrifugal force drift. The parallel acceleration is

$$
a_{\|, s}=-\mu \hat{\mathbf{b}} \cdot \nabla_{\mathbf{X}} B-\frac{Z_{s} e}{m_{s}} \hat{\mathbf{b}} \cdot \nabla_{\mathbf{X}} \Phi_{0}+R \Omega_{\zeta}^{2} \hat{\mathbf{b}} \cdot \nabla_{\mathbf{X}} R .
$$

Finally, $\left\langle\bar{C}_{s s^{\prime}}^{(\ell)}\right\rangle_{s}=\left\langle C_{s s^{\prime}}\left[\bar{h}_{s}\left(\mathbf{x}-\boldsymbol{\rho}_{s}, w_{\|}, \mu, t\right), f_{M s^{\prime}}\right]\right\rangle_{s}+$ $\left\langle C_{s s^{\prime}}\left[f_{M s}, \bar{h}_{s^{\prime}}\left(\mathbf{x}-\boldsymbol{\rho}_{s^{\prime}}, w_{\|}, \mu, t\right)\right]\right\rangle_{s}$ is the gyroaveraged linearized collision operator between species $s$ and $s^{\prime}$. Here $C_{s s^{\prime}}\left[f_{s}, f_{s^{\prime}}\right]$ is the bilinear full collision operator, and we have emphasized that $\bar{h}_{s}\left(\mathbf{X}, w_{\|}, \mu, t\right)$ and $\bar{h}_{s^{\prime}}\left(\mathbf{X}, w_{\|}, \mu, t\right)$ depend on velocity space not only through the variables $w_{\|}$and $\mu$ but also through $\mathbf{X}=\mathbf{x}-\boldsymbol{\rho}_{s}(\mu, \varphi)$. The linearized collision operator is linear in both $\bar{h}_{s}$ and $\bar{h}_{s^{\prime}}$. To show the symmetries of the local $\delta f$ gyrokinetic model, it is convenient to write the collision operator using the Rosenbluth potentials [26]. The particular expressions that we use are given in Appendix A.

The fluctuations of the electrostatic potential are obtained using the quasineutrality equation, $\sum_{s} Z_{s} \int d^{3} w \delta \bar{f}_{s}=0$, where the integral in velocity space is taken at fixed $\mathbf{x}$. Employing $d^{3} w=B d w_{\|} d \mu d \varphi$, we find that quasineutrality becomes

$$
\sum_{s} Z_{s} B \int d w_{\|} d \mu d \varphi \bar{h}_{s}\left(\mathbf{x}-\boldsymbol{\rho}_{s}\right)-\sum_{s} \frac{Z_{s}^{2} e n_{s} \bar{\phi}}{T_{s}}=0
$$

where we have stressed that $\bar{h}_{s}$ depends on velocity not only through $w_{\|}$and $\mu$, but also through $\mathbf{X}=$ $\mathbf{x}-\boldsymbol{\rho}_{s}(\mu, \varphi)$. The parallel vector potential $\bar{A}_{\|}$and the parallel magnetic field $\bar{B}_{\|}$are both determined by the current equation $\nabla \times\left(\nabla \bar{A}_{\|} \times \hat{\mathbf{b}}+\bar{B}_{\| \mid} \hat{\mathbf{b}}\right)=$ $(4 \pi / c) \sum_{s} Z_{s} e \int d^{3} w \delta \bar{f}_{s} \mathbf{w}$. The parallel component of this equation gives

$$
-\nabla_{\perp}^{2} \bar{A}_{\|}=\sum_{s} \frac{4 \pi Z_{s} e B}{c} \int d w_{\|} d \mu d \varphi \bar{h}_{s}\left(\mathbf{x}-\boldsymbol{\rho}_{s}\right) w_{\|}
$$

and the perpendicular component is

$$
\nabla \bar{B}_{||} \times \hat{\mathbf{b}}=\sum_{s} \frac{4 \pi Z_{s} e B}{c} \int d w_{\|} d \mu d \varphi \bar{h}_{s}\left(\mathbf{x}-\boldsymbol{\rho}_{s}\right) \mathbf{w}_{\perp} .
$$

We have neglected the parallel gradient $\hat{\mathbf{b}} \cdot \nabla \sim 1 / a$ of $\bar{A}_{\|}$ and the gradient $\nabla \sim 1 / a$ of the background magnetic field $\mathbf{B}$ because they are much smaller than the perpendicular gradients $\nabla_{\perp} \sim 1 / \rho_{i}$ of the fluctuations.

\section{Local approximation}

Since the characteristic perpendicular length of the turbulent structures is much shorter than the parallel length, it is convenient to describe the turbulent pieces $\bar{h}_{s}\left(\psi(\mathbf{X}), \alpha(\mathbf{X}, t), \theta(\mathbf{X}), w_{\|}, \mu, t\right), \bar{\phi}(\psi(\mathbf{x}), \alpha(\mathbf{x}, t), \theta(\mathbf{x}), t)$, $\bar{A}_{\| \mid}(\psi(\mathbf{x}), \alpha(\mathbf{x}, t), \theta(\mathbf{x}), t)$ and $\bar{B}_{||}(\psi(\mathbf{x}), \alpha(\mathbf{x}, t), \theta(\mathbf{x}), t)$ by two coordinates perpendicular to the magnetic field line, $\psi$ and $\alpha$, and a coordinate to locate the position along the magnetic field that in this case is the poloidal angle $\theta$. We stress that the distribution function depends on the guiding center position $\mathbf{X}$ and that the electromagnetic fields depend on the position $\mathbf{x}$. Here $\alpha(\mathbf{x}, t)$ is the coordinate in the direction perpendicular to the magnetic field within the flux surface and rotating with toroidal angular velocity $\Omega_{\zeta}$, defined such that $\mathbf{B}=\nabla \alpha \times \nabla \psi$ and $\partial \alpha / \partial t+\Omega_{\zeta}(\partial \alpha / \partial \zeta)=0$, i.e.,

$$
\alpha=\zeta-q(\psi) \vartheta(\psi, \theta)-\Omega_{\zeta}(\psi) t,
$$

where

$$
\vartheta(\psi, \theta)=\left.\frac{I(\psi)}{q(\psi)} \int_{0}^{\theta} d \theta^{\prime}\left(R^{2} \mathbf{B} \cdot \nabla \theta\right)^{-1}\right|_{\psi, \theta^{\prime}}
$$


and $q(\psi)=\left|(I / 2 \pi) \int_{0}^{2 \pi} d \theta\left(R^{2} \mathbf{B} \cdot \nabla \theta\right)^{-1}\right|$ is the safety factor. The advantage of using the time dependent variable $\alpha$ is that the $\mathbf{E} \times \mathbf{B}$ shear [11, 16 is included in the formulation in a natural way, as we will see.

In the local approximation, the characteristic perpendicular size of the turbulent structures is assumed to be small compared to the background length scale. The local $\delta f$ equations only depend on $\psi$ and $\alpha$ through the unknowns $\bar{h}_{s}, \bar{\phi}, \bar{A}_{\|}$and $\bar{B}_{\|}$. It is then more convenient to Fourier analyze the distribution function and the electromagnetic fields. Defining $g=$ $(\Delta \psi \Delta \alpha)^{-1} \int_{\Delta \psi} d \psi \int_{\Delta \alpha} d \alpha \bar{g} \exp \left(-i k_{\psi} \psi-i k_{\alpha} \alpha\right)$, with $i=$ $\sqrt{-1}, g=\left\{h_{s}, \phi, A_{\|}, B_{\|}\right\}, \bar{g}=\left\{\bar{h}_{s}, \bar{\phi}, \bar{A}_{\|}, \bar{B}_{\|}\right\}$, and $\Delta \psi$ and $\Delta \alpha$ the size of the domain of interest, and using Appendix $\mathrm{B}$ the $\delta f$ gyrokinetic equation becomes

$$
\begin{array}{r}
\frac{\partial h_{s}}{\partial t}+w_{\|} \hat{\mathbf{b}} \cdot \nabla \theta \frac{\partial h_{s}}{\partial \theta}+i k_{\psi} h_{s} v_{d, s}^{\psi}+i k_{\alpha} h_{s} v_{d, s}^{\alpha} \\
+a_{\|, s} \frac{\partial h_{s}}{\partial w_{\|}}-\sum_{s^{\prime}}\left\langle C_{s s^{\prime}}^{(\ell)}\right\rangle_{s}+\left\{\langle\chi\rangle_{s}, h_{s}\right\} \\
=\frac{Z_{s} e f_{M s}}{T_{s}} \frac{\partial\langle\chi\rangle_{s}}{\partial t}-f_{M s} v_{\chi, s}^{\psi}\left[\frac{1}{n_{s}} \frac{\partial n_{s}}{\partial \psi}+\frac{m_{s} I w_{\|}}{B T_{s}} \frac{\partial \Omega_{\zeta}}{\partial \psi}\right. \\
\left.+\frac{Z_{s} e}{T_{s}} \frac{\partial \Phi_{0}}{\partial \psi}-\frac{m_{s} R \Omega_{\zeta}^{2}}{T_{s}} \frac{\partial R}{\partial \psi}+\left(\frac{m_{s} w^{2}}{2 T_{s}}-\frac{3}{2}\right) \frac{1}{T_{s}} \frac{\partial T_{s}}{\partial \psi}\right]
\end{array}
$$

Here, the turbulent components of the electromagnetic field are contained in

$$
\langle\chi\rangle_{s}=J_{0}\left(z_{s}\right)\left(\phi-\frac{1}{c} w_{\|} A_{\|}\right)+\frac{2 J_{1}\left(z_{s}\right)}{z_{s}} \frac{m_{s} \mu}{Z_{s} e} B_{\|},
$$

where $J_{n}\left(z_{s}\right)$ is the $n$-th Bessel function of the first kind. The function $z_{s}\left(k_{\psi}, k_{\alpha}, \theta, \mu, t\right)$ is

$$
z_{s}\left(k_{\psi}, k_{\alpha}, \theta, \mu, t\right)=\frac{k_{\perp} \sqrt{2 \mu B}}{\Omega_{s}},
$$

with

$$
k_{\perp}=\left(k_{\psi}^{2}|\nabla \psi|^{2}+2 k_{\psi} k_{\alpha} \nabla \psi \cdot \nabla \alpha+k_{\alpha}^{2}|\nabla \alpha|^{2}\right)^{1 / 2}
$$

the magnitude of the perpendicular wavenumber. Note that the perpendicular wavenumber increases with time because $\nabla \alpha=\ldots-\nabla \psi\left(\partial \Omega_{\zeta} / \partial \psi\right) t$. This is equivalent to the procedure generally employed in local $\delta f$ simulations to account for the shear in the $\mathbf{E} \times \mathbf{B}$ drift [10, 11, 27]. The generalized potential $\langle\chi\rangle_{s}$ appears in the radial turbulent drift

$$
v_{\chi, s}^{\psi}=i k_{\alpha} c\langle\chi\rangle_{s}
$$

and in the nonlinear term

$$
\begin{array}{r}
\left\{\langle\chi\rangle_{s}, h_{s}\right\}=c \sum_{k_{\psi}^{\prime}, k_{\alpha}^{\prime}}\left(k_{\psi}^{\prime} k_{\alpha}-k_{\psi} k_{\alpha}^{\prime}\right)\langle\chi\rangle_{s}\left(k_{\psi}^{\prime}, k_{\alpha}^{\prime}\right) \\
\times h_{s}\left(k_{\psi}-k_{\psi}^{\prime}, k_{\alpha}-k_{\alpha}^{\prime}\right) .
\end{array}
$$

The $\psi$ and $\alpha$ components of the drift velocity are

$$
\begin{aligned}
& v_{d, s}^{\psi}=\left[-\frac{c I}{B} \frac{\partial \Phi_{0}}{\partial \theta}-\frac{I\left(w_{\|}^{2}+\mu B\right)}{B \Omega_{s}} \frac{\partial B}{\partial \theta}\right. \\
& \left.+\frac{2 B R \Omega_{\zeta} w_{\|}}{\Omega_{s}} \frac{\partial R}{\partial \theta}+\frac{I R \Omega_{\zeta}^{2}}{\Omega_{s}} \frac{\partial R}{\partial \theta}\right] \hat{\mathbf{b}} \cdot \nabla \theta
\end{aligned}
$$

and

$$
\begin{gathered}
v_{d, s}^{\alpha}=-c\left[\frac{\partial \Phi_{0}}{\partial \psi}-\frac{\partial \Phi_{0}}{\partial \theta} \frac{(\nabla \alpha \times \hat{\mathbf{b}}) \cdot \nabla \theta}{B}\right] \\
-\frac{w_{\|}^{2}+\mu B}{\Omega_{s}}\left[\frac{\partial B}{\partial \psi}-\frac{\partial B}{\partial \theta} \frac{(\nabla \alpha \times \hat{\mathbf{b}}) \cdot \nabla \theta}{B}\right] \\
-\left.\frac{4 \pi w_{\|}^{2}}{B \Omega_{s}} \frac{\partial p}{\partial \psi}\right|_{R}+\frac{2 \Omega_{\zeta} w_{\|}}{\Omega_{s}}(\nabla R \times \hat{\boldsymbol{\zeta}}) \cdot \nabla \alpha \\
+\frac{m_{s} c R \Omega_{\zeta}^{2}}{Z_{s} e}\left[\frac{\partial R}{\partial \psi}-\frac{\partial R}{\partial \theta} \frac{(\nabla \alpha \times \hat{\mathbf{b}}) \cdot \nabla \theta}{B}\right] .
\end{gathered}
$$

The parallel acceleration is

$$
a_{\|, s}=\left(-\mu \frac{\partial B}{\partial \theta}-\frac{Z_{s} e}{m_{s}} \frac{\partial \Phi_{0}}{\partial \theta}+R \Omega_{\zeta}^{2} \frac{\partial R}{\partial \theta}\right) \hat{\mathbf{b}} \cdot \nabla \theta .
$$

The Fourier transform of the gyroaveraged collision operator for collisions between species $s$ and $s^{\prime},\left\langle C_{s s^{\prime}}^{(\ell)}\right\rangle_{s}$, is given in Appendix C.

The local versions of the quasineutrality equation (14), the parallel current equation (15) and the perpendicular current equation (16) are obtained in Appendix D, and are given by

$$
\sum_{s} 2 \pi Z_{s} B \int d w_{\|} d \mu h_{s} J_{0}\left(z_{s}\right)-\sum_{s} \frac{Z_{s}^{2} e n_{s} \phi}{T_{s}}=0,
$$

$$
k_{\perp}^{2} A_{\|}=\sum_{s} \frac{8 \pi^{2} Z_{s} e B}{c} \int d w_{\|} d \mu h_{s} w_{\|} J_{0}\left(z_{s}\right)
$$

and

$$
\frac{B B_{\|}}{4 \pi}+\sum_{s} 2 \pi m_{s} B^{2} \int d w_{\|} d \mu h_{s} \mu \frac{2 J_{1}\left(z_{s}\right)}{z_{s}}=0 .
$$

The perpendicular current equation 30 has been rewritten as a perpendicular pressure balance.

\section{Radial flux of toroidal angular momentum} by

The radial flux of toroidal angular momentum is given

$$
\Pi=\sum_{s}\left\langle\Pi_{s}\right\rangle_{\Delta t}+\left\langle\Pi_{B}\right\rangle_{\Delta t},
$$


where

$$
\Pi_{s}=m_{s}\left\langle\left\langle R \int d^{3} w f_{s}(\mathbf{v} \cdot \hat{\boldsymbol{\zeta}})(\mathbf{v} \cdot \nabla \psi)\right\rangle_{\psi}\right\rangle_{\Delta \psi}
$$

is the momentum flux due to the species $s$, and

$$
\Pi_{B}=-\frac{1}{4 \pi}\left\langle\langle R[(\mathbf{B}+\delta \overline{\mathbf{B}}) \cdot \hat{\boldsymbol{\zeta}}][(\mathbf{B}+\delta \overline{\mathbf{B}}) \cdot \nabla \psi]\rangle_{\psi}\right\rangle_{\Delta \psi}
$$

is the momentum flux due to the Maxwell stress. Here, $\langle\ldots\rangle_{\psi}=\left(V^{\prime}\right)^{-1} \int d \theta d \zeta \mathcal{J}(\ldots)$ is the flux surface average, $V^{\prime} \equiv d V / d \psi=2 \pi \int d \theta \mathcal{J}$, and $\langle\ldots\rangle_{\Delta \psi}=$ $\Delta \psi^{-1} \int_{\Delta \psi} d \psi(\ldots)$ and $\langle\ldots\rangle_{\Delta t}=\Delta t^{-1} \int_{\Delta t} d t(\ldots)$ are coarse grain averages over a radial region $\Delta \psi$ and over a time interval $\Delta t$ larger than the decorrelation length and time of the turbulence, respectively. In the local approximation, $\Delta \psi$ is much shorter than the characteristic length of variation of the background profiles of density, temperature and velocity, and $\Delta t$ is much shorter than the transport time scale. We remind the reader that the velocity $\mathbf{v}=\mathbf{w}+R \Omega_{\zeta} \hat{\boldsymbol{\zeta}}$, used in equation (32) and in some other equations in this section, is the velocity in the laboratory frame. Note also that the distribution function $f_{s}$ includes the lowest order Maxwellian $f_{M s}$, the turbulent contribution $\delta \bar{f}_{s}$ and neoclassical higher order pieces. Finally, it is worth pointing out that our definition of radial flux of toroidal angular momentum does not have units of pressure $\times$ length, but of pressure $\times(\text { length })^{2} \times($ magnetic field $)$ because our radial variable $\psi$, which appears in the gradient $\nabla \psi$, has dimensions of (length $)^{2} \times($ magnetic field $)$.

To calculate an expression for the turbulent contribution to $\Pi_{s}$, it is convenient to use the full Fokker-Planck equation

$$
\begin{array}{r}
\frac{\partial f_{s}}{\partial t}+\mathbf{v} \cdot \nabla f_{s}+\left\{\Omega_{s} \mathbf{v} \times \hat{\mathbf{b}}+\frac{Z_{s} e}{m_{s}}\left[-\nabla\left(\Phi_{-1}+\Phi_{0}\right)\right.\right. \\
\left.\left.+\delta \overline{\mathbf{E}}+\frac{1}{c} \mathbf{w} \times \delta \overline{\mathbf{B}}\right]\right\} \cdot \nabla_{v} f_{s}=\sum_{s^{\prime}} C_{s s^{\prime}}\left[f_{s}, f_{s^{\prime}}\right] .
\end{array}
$$

The turbulent Lorentz force is expressed in terms of the peculiar velocity $\mathbf{w}=\mathbf{v}-R \Omega_{\zeta} \hat{\boldsymbol{\zeta}}$ because the fluctuating magnetic fields are calculated in the rotating frame. By taking moments of (34) we obtain a simplified expression for $\Pi_{s}\left[17,28\right.$. The $m_{s} R^{2}(\mathbf{v} \cdot \hat{\boldsymbol{\zeta}})^{2}$ moment gives

$$
\begin{array}{r}
\frac{2 Z_{s} e R}{c} \int d^{3} w f_{s}(\mathbf{v} \cdot \hat{\boldsymbol{\zeta}})(\mathbf{v} \cdot \nabla \psi)=-2 Z_{s} e R^{2} \int d^{3} w f_{s}(\mathbf{v} \cdot \hat{\boldsymbol{\zeta}})\left(\delta \overline{\mathbf{E}}+\frac{1}{c} \mathbf{w} \times \delta \overline{\mathbf{B}}\right) \cdot \hat{\boldsymbol{\zeta}}+\frac{\partial}{\partial t}\left[m_{s} R^{2} \int d^{3} w f_{s}(\mathbf{v} \cdot \hat{\boldsymbol{\zeta}})^{2}\right] \\
+\nabla \cdot\left[m_{s} R^{2} \int d^{3} w f_{s} \mathbf{v}(\mathbf{v} \cdot \hat{\boldsymbol{\zeta}})^{2}\right]-m_{s} R^{2} \sum_{s^{\prime}} \int d^{3} w C_{s s^{\prime}}\left[f_{s}, f_{s^{\prime}}\right](\mathbf{v} \cdot \hat{\boldsymbol{\zeta}})^{2}
\end{array}
$$

where we have used $R \mathbf{B} \times \hat{\boldsymbol{\zeta}}=\nabla \psi$ and $\nabla(R \hat{\boldsymbol{\zeta}})=(\nabla R) \hat{\boldsymbol{\zeta}}-\hat{\boldsymbol{\zeta}}(\nabla R)$. Taking a flux surface average and the coarse grain averages over radius and time in this equation, we find that the second and third terms in the right side vanish to order $\epsilon_{i}^{2} p_{s} R|\nabla \psi|$. The last term only gives a neoclassical contribution, leaving the turbulent transport of momentum

$$
\left\langle\Pi_{s}^{\mathrm{tb}}\right\rangle_{\Delta t}=-c\left\langle\left\langle\left\langle m_{s} R^{2} \int d^{3} w \bar{h}_{s}\left(\mathbf{w} \cdot \hat{\boldsymbol{\zeta}}+R \Omega_{\zeta}\right)\left(\delta \overline{\mathbf{E}}+\frac{1}{c} \mathbf{w} \times \delta \overline{\mathbf{B}}\right) \cdot \hat{\boldsymbol{\zeta}}\right\rangle_{\psi}\right\rangle_{\Delta \psi}\right\rangle_{\Delta t} \sim \epsilon_{i}^{2} p_{s} R|\nabla \psi| .
$$

Note that only the fluctuating piece of the distribution function $\delta \bar{f}_{s}=\bar{h}_{s}-\left(Z_{s} e \bar{\phi} / T_{s}\right) f_{M s}$ contributes to the turbulent transport of toroidal angular momentum because the coarse grain averages satisfy $\left\langle\left\langle\delta \bar{f}_{s}\right\rangle_{\Delta \psi}\right\rangle_{\Delta t}=0$, $\left\langle\langle\delta \overline{\mathbf{E}}\rangle_{\Delta \psi}\right\rangle_{\Delta t}=0$ and $\left\langle\langle\delta \overline{\mathbf{B}}\rangle_{\Delta \psi}\right\rangle_{\Delta t}=0$. In addition, the contribution from the Maxwell-Boltzmann response $-\left(Z_{s} e \bar{\phi} / T_{s}\right) f_{M s}$ vanishes upon integrating over velocity space and over the spatial scales of the turbulence.

The turbulent contribution to the Maxwell stress is

$$
\Pi_{B}^{\mathrm{tb}}=-\frac{1}{4 \pi}\left\langle\langle R(\delta \overline{\mathbf{B}} \cdot \hat{\boldsymbol{\zeta}})(\delta \overline{\mathbf{B}} \cdot \nabla \psi)\rangle_{\psi}\right\rangle_{\Delta \psi} .
$$

This contribution is usually small for low $\beta$ plasmas, but it can become important where the pressure gradient ap- proaches the ballooning threshold [29, 30].

To study the different contributions to the turbulent transport of momentum in 36 and 37 , we rewrite $\Pi_{s}^{\text {tb }}$ and $\Pi_{B}^{\text {tb }}$ using $\delta \overline{\mathbf{E}}=-\nabla_{\perp} \bar{\phi}, \delta \overline{\mathbf{B}}=\bar{B}_{\|} \hat{\mathbf{b}}+\nabla \bar{A}_{\|} \times \hat{\mathbf{b}}$, $\mathbf{w} \times \delta \overline{\mathbf{B}}=\nabla_{\perp} \bar{A}_{\|} w_{\|}-\left(\mathbf{w}_{\perp} \cdot \nabla_{\perp} \bar{A}_{\|}\right) \hat{\mathbf{b}}+\bar{B}_{\| \mid}(\mathbf{w} \times \hat{\mathbf{b}})$ and $R \hat{\boldsymbol{\zeta}}=(I \hat{\mathbf{b}} / B)-(\hat{\mathbf{b}} \times \nabla \psi / B)$, giving

$$
\Pi_{s}^{\mathrm{tb}}=\pi_{s, \|}+\pi_{s, \perp}+m_{s}\left\langle R^{2}\right\rangle_{\psi} \Omega_{\zeta} g_{s}
$$

and

$$
\Pi_{B}^{\mathrm{tb}}=\pi_{B, \|}+\pi_{B, \perp} .
$$

The transport of momentum has three contributions: a piece $\sum_{s} m_{s}\left\langle R^{2}\right\rangle_{\psi} \Omega_{\zeta} g_{s}$, where $g_{s}$ is very similar to 
but not exactly the radial flux of particles, a piece $\sum_{s}\left(\pi_{s, \|}+\pi_{s, \perp}\right)$ that is related to the radial-toroidal component of the viscosity and the Reynolds stress, and the Maxwell stress $\pi_{B, \|}+\pi_{B, \perp}$. The different contributions $\pi_{s, \|}, \pi_{s, \perp}, \pi_{B, \|}$ and $\pi_{B, \perp}$ are classified according to the component of the momentum that they are transporting. The components

$$
\begin{aligned}
\pi_{s, \|}= & \left\langle\left\langle\frac { m _ { s } I } { B } \int d ^ { 3 } w \overline { h } _ { s } \left\{ w_{\|} \bar{v}_{\mathrm{tb}}^{\psi}+\frac{1}{B}\left[I w_{\|}\right.\right.\right.\right. \\
& \left.\left.\left.-(\mathbf{w} \times \hat{\mathbf{b}}) \cdot \nabla \psi]\left(\mathbf{w}_{\perp} \cdot \nabla \bar{A}_{\| \mid}\right)\right\}\right\rangle_{\psi}\right\rangle_{\Delta \psi}
\end{aligned}
$$

and

$$
\pi_{B, \|}=-\left\langle\left\langle\frac{I}{4 \pi B} \bar{B}_{\|}\left(\nabla \bar{A}_{\|} \times \hat{\mathbf{b}}\right) \cdot \nabla \psi\right\rangle_{\psi}\right\rangle_{\Delta \psi}
$$

transport parallel momentum and dominate in a tokamak where the magnetic field is mainly toroidal. Here the radial component of the turbulent velocity is

$$
\bar{v}_{\mathrm{tb}}^{\psi}=\left(-\frac{c}{B} \nabla \bar{\phi} \times \hat{\mathbf{b}}+\frac{w_{\|}}{B} \nabla \bar{A}_{\|} \times \hat{\mathbf{b}}-\frac{\bar{B}_{\|}}{B} \mathbf{w}_{\perp}\right) \cdot \nabla \psi .
$$

The components

$$
\pi_{s, \perp}=-\left\langle\left\langle\frac{m_{s}}{B} \int d^{3} w \bar{h}_{s}[(\mathbf{w} \times \hat{\mathbf{b}}) \cdot \nabla \psi] \bar{v}_{\mathrm{tb}}^{\psi}\right\rangle_{\psi}\right\rangle_{\Delta \psi}
$$

and

$$
\pi_{B, \perp}=-\left\langle\left\langle\frac{1}{4 \pi B}\left(\nabla \bar{A}_{\|} \cdot \nabla \psi\right)\left(\nabla \bar{A}_{\|} \times \hat{\mathbf{b}}\right) \cdot \nabla \psi\right\rangle_{\psi}\right\rangle_{\Delta \psi}
$$

transport perpendicular momentum and dominate for tokamaks where the magnetic field is mainly poloidal. Finally, $g_{s}$ is

$$
g_{s}=\frac{1}{\left\langle R^{2}\right\rangle_{\psi}}\left\langle\left\langle R^{2} \int d^{3} w \bar{h}_{s} \bar{v}_{\mathrm{tb}}^{\psi}\right\rangle_{\psi}\right\rangle_{\Delta \psi} .
$$

In Appendix E we evaluate $\pi_{s, \|}, \pi_{s, \perp}, g_{s}, \pi_{B, \|}$ and $\pi_{B, \perp}$ employing the Fourier analyzed distribution function and electromagnetic field obtained from the local formulation in section IIC. In what follows, the different contributions to $\pi_{s, \|}, \pi_{s, \perp}$ and $g_{s}$ are classified according to which type of fluctuation in the electromagnetic fields drives them. The flux of parallel momentum due to species $s$ is $\pi_{s, \|}=\pi_{s, \|}^{\phi}+\pi_{s, \|}^{A_{\|}}+\pi_{s, \|}^{B_{\|}}$, with

$$
\begin{aligned}
\pi_{s, \|}^{\phi}= & \frac{4 \pi^{2} i m_{s} c I}{V^{\prime}} \sum_{k_{\psi}, k_{\alpha}} k_{\alpha} \int d \theta \mathcal{J} \phi\left(k_{\psi}, k_{\alpha}\right) \\
& \times \int d w_{\|} d \mu h_{s}\left(-k_{\psi},-k_{\alpha}\right) w_{\|} J_{0}\left(z_{s}\right),
\end{aligned}
$$

$$
\begin{array}{r}
\pi_{s, \|}^{A_{\|}}=-\frac{4 \pi^{2} i m_{s} I}{V^{\prime}} \sum_{k_{\psi}, k_{\alpha}} k_{\alpha} \int d \theta \mathcal{J} A_{\|}\left(k_{\psi}, k_{\alpha}\right) \\
\times \int d w_{\|} d \mu h_{s}\left(-k_{\psi},-k_{\alpha}\right)\left[w_{\|}^{2} J_{0}\left(z_{s}\right)-\mu B \frac{2 J_{1}\left(z_{s}\right)}{z_{s}}\right]
\end{array}
$$

and

$$
\begin{aligned}
\pi_{s, \|}^{B_{\|}} & =\frac{4 \pi^{2} i m_{s}^{2} c I}{Z_{s} e V^{\prime}} \sum_{k_{\psi}, k_{\alpha}} k_{\alpha} \int d \theta \mathcal{J} B_{\|}\left(k_{\psi}, k_{\alpha}\right) \\
& \times \int d w_{\|} d \mu h_{s}\left(-k_{\psi},-k_{\alpha}\right) w_{\|} \mu \frac{2 J_{1}\left(z_{s}\right)}{z_{s}} .
\end{aligned}
$$

Similarly $\pi_{s, \perp}=\pi_{s, \perp}^{\phi}+\pi_{s, \perp}^{A_{\|}}+\pi_{s, \perp}^{B_{\|}}$, with

$$
\begin{aligned}
\pi_{s, \perp}^{\phi}= & -\frac{4 \pi^{2} m_{s}^{2} c^{2}}{Z_{s} e V^{\prime}} \sum_{k_{\psi}, k_{\alpha}} k_{\alpha} \int d \theta \mathcal{J} k^{\psi} \phi\left(k_{\psi}, k_{\alpha}\right) \\
& \times \int d w_{\|} d \mu h_{s}\left(-k_{\psi},-k_{\alpha}\right) \mu \frac{2 J_{1}\left(z_{s}\right)}{z_{s}} \\
\pi_{s, \perp}^{A_{\|}}= & \frac{4 \pi^{2} m_{s}^{2} c}{Z_{s} e V^{\prime}} \sum_{k_{\psi}, k_{\alpha}} k_{\alpha} \int d \theta \mathcal{J} k^{\psi} A_{\|}\left(k_{\psi}, k_{\alpha}\right) \\
& \times \int d w_{\|} d \mu h_{s}\left(-k_{\psi},-k_{\alpha}\right) w_{\|} \mu \frac{2 J_{1}\left(z_{s}\right)}{z_{s}}
\end{aligned}
$$

and

$$
\begin{array}{r}
\pi_{s, \perp}^{B_{\|}}=-\frac{2 \pi^{2} m_{s}^{3} c^{2}}{Z_{s}^{2} e^{2} V^{\prime}} \sum_{k_{\psi}, k_{\alpha}} k_{\alpha} \int d \theta \mathcal{J} k^{\psi} B_{\|}\left(k_{\psi}, k_{\alpha}\right) \\
\times \int d w_{\|} d \mu h_{s}\left(-k_{\psi},-k_{\alpha}\right) \mu^{2} G\left(z_{s}\right),
\end{array}
$$

where $G\left(z_{s}\right)=\left[8 J_{1}\left(z_{s}\right)+4 z_{s} J_{2}\left(z_{s}\right)-4 z_{s} J_{0}\left(z_{s}\right)\right] / z_{s}^{3}$ and $k^{\psi}=\mathbf{k}_{\perp} \cdot \nabla \psi=k_{\psi}|\nabla \psi|^{2}+k_{\alpha} \nabla \psi \cdot \nabla \alpha$ is the projection of the wavevector on the radial direction. The component $g_{s}=g_{s}^{\phi}+g_{s}^{A_{\|}}+g_{s}^{B_{\|}}$, where

$$
\begin{aligned}
& g_{s}^{\phi}=\frac{4 \pi^{2} i c}{V^{\prime}\left\langle R^{2}\right\rangle_{\psi}} \sum_{k_{\psi}, k_{\alpha}} k_{\alpha} \int d \theta \mathcal{J} R^{2} B \phi\left(k_{\psi}, k_{\alpha}\right) \\
& \times \int d w_{\|} d \mu h_{s}\left(-k_{\psi},-k_{\alpha}\right) J_{0}\left(z_{s}\right), \\
& g_{s}^{A_{\|}}=-\frac{4 \pi^{2} i}{V^{\prime}\left\langle R^{2}\right\rangle_{\psi}} \sum_{k_{\psi}, k_{\alpha}} k_{\alpha} \int d \theta \mathcal{J} R^{2} B A_{\|}\left(k_{\psi}, k_{\alpha}\right) \\
& \times \int d w_{\|} d \mu h_{s}\left(-k_{\psi},-k_{\alpha}\right) w_{\|} J_{0}\left(z_{s}\right)
\end{aligned}
$$

and

$$
\begin{array}{r}
g_{s}^{B_{\|}}=\frac{4 \pi^{2} i m_{s} c}{Z_{s} e V^{\prime}\left\langle R^{2}\right\rangle_{\psi}} \sum_{k_{\psi}, k_{\alpha}} k_{\alpha} \int d \theta \mathcal{J} R^{2} B B_{\|}\left(k_{\psi}, k_{\alpha}\right) \\
\quad \times \int d w_{\|} d \mu h_{s}\left(-k_{\psi},-k_{\alpha}\right) \mu \frac{2 J_{1}\left(z_{s}\right)}{z_{s}}
\end{array}
$$


(a)

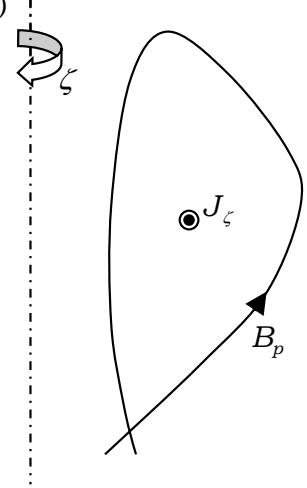

(b)

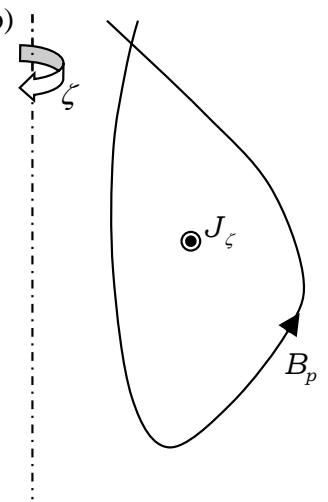

FIG. 1: Equilibrium (a) and its up-down reflection (b). The dash-dot line is the axis of symmetry.

The Maxwell stress contribution to the transport of parallel momentum is

$$
\pi_{B, \|}=-\frac{i I}{2 V^{\prime}} \sum_{k_{\psi}, k_{\alpha}} k_{\alpha} \int d \theta \mathcal{J} B_{\|}\left(k_{\psi}, k_{\alpha}\right) A_{\|}\left(-k_{\psi},-k_{\alpha}\right) .
$$

Finally, the Maxwell stress contribution to the transport of perpendicular momentum is

$$
\pi_{B, \perp}=\frac{1}{2 V^{\prime}} \sum_{k_{\psi}, k_{\alpha}} k_{\alpha} \int d \theta \mathcal{J} k^{\psi}\left|A_{\|}\left(k_{\psi}, k_{\alpha}\right)\right|^{2} .
$$

\section{SYMMETRY OF THE LOCAL $\delta f$ GYROKINETIC FORMULATION}

In this section we present two symmetries of the local $\delta f$ gyrokinetic formulation. Both of them involve an updown reflection as represented in Fig. 1. Note that the shape of the flux surface is an up-down reflection of the original one, but the direction of the poloidal magnetic field is unchanged. Thus, this reflection leaves the direction of the toroidal plasma current unchanged. This up-down reflection does not affect the toroidal magnetic field. In fact, one of the symmetries that we present here will leave it unchanged whereas the other reverses it. Importantly, for a given equilibrium, with some chosen poloidal angle definition $\theta$, it is possible to find a related poloidal angle $\theta$ for the up-down reflection such that the functions of $\psi$ and $\theta$ given in Table $\mathbb{1}$ satisfy the relations given in that Table. We prove this in Appendix F

There are two transformations, which we will call transformations I and II, that leave the local $\delta f$ gyrokinetic system of equations unchanged. They are given in Table II. To prove that these symmetries hold, it is necessary to know how certain coefficients in the gyrokinetic equations transform under up-down reflection. This

TABLE I: Equilibrium quantities under up-down reflection

\begin{tabular}{ll}
\hline \hline Before up-down reflection & After up-down reflection \\
\hline$|\nabla \psi|^{2}, \nabla \psi \cdot \nabla \theta,|\nabla \theta|^{2}, B$ & $|\nabla \psi|^{2}, \nabla \psi \cdot \nabla \theta,|\nabla \theta|^{2}, B$, \\
$\mathcal{J}, n_{s}, \Phi_{0}, p, \partial p /\left.\partial \psi\right|_{R}$ & $\mathcal{J}, n_{s}, \Phi_{0}, p, \partial p /\left.\partial \psi\right|_{R}$ \\
$\mathbf{B} \cdot \nabla \theta$ & $-\mathbf{B} \cdot \nabla \theta$ \\
\hline
\end{tabular}

TABLE II: Transformation I and II that leave the local $\delta f$ gyrokinetic system of equations unchanged.

\begin{tabular}{lll}
\hline \hline & Before transformation & After transformation \\
\hline & Geometry & Up-down reflected geometry \\
I & $\mu, k_{\alpha}, A_{\|}$ & $\mu, k_{\alpha}, A_{\|}$ \\
& $\Omega_{\zeta}, \partial \Omega_{\zeta} / \partial \psi, w_{\|}, k_{\psi}$, & $-\Omega_{\zeta},-\partial \Omega_{\zeta} / \partial \psi,-w_{\|},-k_{\psi}$, \\
& $h_{s}, \phi, B_{\|}$ & $-h_{s},-\phi,-B_{\|}$ \\
\hline \multirow{2}{*}{ II Geometry } & Up-down reflected geometry \\
& $\mu, k_{\psi}, k_{\alpha}, h_{s}, \phi, B_{\|}$ & $\mu, k_{\psi}, k_{\alpha}, h_{s}, \phi, B_{\|}$ \\
& $I, w_{\|}, A_{\|}$ & $-I,-w_{\|},-A_{\|}$ \\
\hline \hline
\end{tabular}

information is contained in Tables I and III Table III. derived in Appendix G, is for the coefficients that contain $\nabla \alpha$. Since they depend on $I$ and $\partial \Omega_{\zeta} / \partial \psi$, they change differently under transformation I or II. With the information in Tables I and III, it is tedious but straightforward to show that the formulation given in subsection IIC is invariant under the transformations in Table II. The symmetry under transformation I is the one that is closer to the the symmetry discovered in Ref. [15]. Since in Ref. 15 some of the effects that we are considering here were not treated, the transformation is not exactly the same. In particular, Ref. [15] neglected $A_{\|}$ and $B_{\|}$and proposed a transformation composed only of the up-down reflection and the change $w_{\|} \rightarrow-w_{\|}$. This symmetry is only valid for the linearized equations and for the particular case $k_{\psi}=0$, which is the most commonly studied in local $\delta f$ gyrokinetic linear theory. In addition, Ref. 15 did not state that $h_{s}$ and $\phi$ should change sign under the transformation. The reason is that the symmetry of the linearized equations cannot determine if $h_{s}, \phi, A_{\|}$or $B_{\|}$must change sign. Only the nonlinear term can determine that.

\section{CONSEQUENCES FOR THE TRANSPORT OF TOROIDAL ANGULAR MOMENTUM}

According to transformation I in Table II if $h_{s}\left(k_{\psi}, k_{\alpha}, \theta, w_{\|}, \mu, t\right), \quad \phi\left(k_{\psi}, k_{\alpha}, \theta, t\right), \quad A_{\|}\left(k_{\psi}, k_{\alpha}, \theta, t\right)$ and $B_{\|}\left(k_{\psi}, k_{\alpha}, \theta, t\right)$ are solutions of the equations in subsection IIC in a given equilibrium, then $\quad-h_{s}\left(-k_{\psi}, k_{\alpha}, \theta,-w_{\|}, \mu, t\right), \quad-\phi\left(-k_{\psi}, k_{\alpha}, \theta, t\right)$, $A_{\|}\left(-k_{\psi}, k_{\alpha}, \theta, t\right)$ and $-B_{\|}\left(-k_{\psi}, k_{\alpha}, \theta, t\right)$ are solutions in the up-down reflection with $\Omega_{\zeta} \rightarrow-\Omega_{\zeta}$ and $\partial \Omega_{\zeta} / \partial \psi \rightarrow-\partial \Omega_{\zeta} / \partial \psi$. This is important for transport of momentum because the radial transport of toroidal angular momentum, given in subsection IID changes 
TABLE III: Changes under transformations I and II.

\begin{tabular}{|c|c|c|}
\hline & Before transformation & After transformation \\
\hline \multirow[t]{2}{*}{ I } & $\begin{array}{l}|\nabla \alpha|^{2},(\nabla \alpha \times \mathbf{B}) \cdot \nabla \theta \\
(\nabla R \times \hat{\boldsymbol{\zeta}}) \cdot \nabla \alpha\end{array}$ & $\begin{array}{l}|\nabla \alpha|^{2},(\nabla \alpha \times \mathbf{B}) \cdot \nabla \theta \\
(\nabla R \times \hat{\boldsymbol{\zeta}}) \cdot \nabla \alpha\end{array}$ \\
\hline & $\nabla \psi \cdot \nabla \alpha, \nabla \theta \cdot \nabla \alpha$ & $-\nabla \psi \cdot \nabla \alpha,-\nabla \theta \cdot \nabla \alpha$ \\
\hline \multirow[t]{2}{*}{ II } & $\begin{array}{l}\nabla \psi \cdot \nabla \alpha, \nabla \theta \cdot \nabla \alpha,|\nabla \alpha|^{2} \\
(\nabla \alpha \times \mathbf{B}) \cdot \nabla \theta\end{array}$ & $\begin{array}{l}\nabla \psi \cdot \nabla \alpha, \nabla \theta \cdot \nabla \alpha,|\nabla \alpha|^{2} \\
(\nabla \alpha \times \mathbf{B}) \cdot \nabla \theta\end{array}$ \\
\hline & $(\nabla R \times \hat{\boldsymbol{\zeta}}) \cdot \nabla \alpha$ & $-(\nabla R \times \hat{\boldsymbol{\zeta}}) \cdot \nabla \alpha$ \\
\hline
\end{tabular}

sign under transformation I. The contributions $\pi_{s, \|}^{\phi}$, $\pi_{s, \|}^{A_{\|}}, \pi_{s, \|}^{B_{\|}}, \pi_{s, \perp}^{\phi}, \pi_{s, \perp}^{A_{\|}}, \pi_{s, \perp}^{B_{\|}}, \pi_{B, \|}$ and $\pi_{B, \perp}$ change sign. The contributions $g_{s}^{\phi}, g_{s}^{A_{\|}}$and $g_{s}^{B_{\|}}$remain the same, but since they appear in the momentum flux multiplied by $\Omega_{\zeta}$ their total contribution also changes sign. Thus, for any initial condition $h_{s}\left(k_{\psi}, k_{\alpha}, \theta, w_{\|}, \mu, t=0\right)$, there is another initial condition $-h_{s}\left(-k_{\psi}, k_{\alpha}, \theta,-w_{\|}, \mu, t=0\right)$ that gives the opposite $\pi_{s, \|}, \pi_{s, \perp}, \pi_{B, \|}$ and $\pi_{B, \perp}$ and the same $g_{s}$ for the up-down reflection with $\Omega_{\zeta} \rightarrow-\Omega_{\zeta}$ and $\partial \Omega_{\zeta} / \partial \psi \rightarrow-\partial \Omega_{\zeta} / \partial \psi$. Since $\pi_{s, \|}, \pi_{s, \perp}, g_{s}, \pi_{B, \|}$ and $\pi_{B, \perp}$ are time averaged over times longer than the decorrelation time, they are independent of the initial condition, and the radial transport of momentum must change sign under up-down reflection and the change $\Omega_{\zeta} \rightarrow-\Omega_{\zeta}$ and $\partial \Omega_{\zeta} / \partial \psi \rightarrow-\partial \Omega_{\zeta} / \partial \psi$. Because the symmetry changes the sign of each contribution $\pi_{s, \|}^{\phi}, \pi_{s, \|}^{A_{\|}}$, $\pi_{s, \|}^{B_{\|}}, \pi_{s, \perp}^{\phi}, \pi_{s, \perp}^{A_{\|}}, \pi_{s, \perp}^{B_{\|}}, \pi_{B, \|}$ and $\pi_{B, \perp}$ independently, this property holds for each one of them independently. The same argument is valid for $g_{s}^{\phi}, g_{s}^{A_{\|}}$and $g_{s}^{B_{\|}}$. In the particular case of up-down symmetric tokamaks, this symmetry implies that the sign of the radial transport of momentum changes when the sign of $\Omega_{\zeta}$ and $\partial \Omega_{\zeta} / \partial \psi$ is changed, and when $\Omega_{\zeta}=0$ and $\partial \Omega_{\zeta} / \partial \psi=0$, it must vanish. A consequence of this result is that reversing the momentum input in up-down symmetric tokamaks exactly reverses the rotation profile $\Omega_{\zeta}(\psi)$.

For up-down asymmetric configurations, there is turbulent transport of momentum even for $\Omega_{\zeta}=0$ and $\partial \Omega_{\zeta} / \partial \psi=0$ [9]. This momentum flux is strongly dependent on the temperature and density gradients that drive the turbulence. An explicit expression for the turbulent momentum flux due to up-down asymmetry has not been obtained, but it is possible to comment on the size of this contribution for small up-down asymmetry. We distinguish two types of equilibrium quantities that enter in the coefficients of the gyrokinetic system of equations in subsection II C the quantities $Q(\psi, \theta)$ for which, in an up-down symmetric configuration, there exists a poloidal angle definition $\theta$ such that $Q(\psi, \theta)=Q(\psi,-\theta)$, and the quantities $\bar{Q}(\psi, \theta)$, for which $\bar{Q}(\psi, \theta)=-\bar{Q}(\psi,-\theta)$. The former quantities are $Q(\psi, \theta)=\left\{|\nabla \psi|^{2},|\nabla \theta|^{2}, B, \mathbf{B}\right.$. $\left.\nabla \theta, \mathcal{J}, n_{s}, \Phi_{0}, p, \partial p /\left.\partial \psi\right|_{R}\right\}$ and the latter quantities are $\bar{Q}(\psi, \theta)=\{\nabla \psi \cdot \nabla \theta\}$. Any equilibrium quantity can be divided in its up-down symmetric and antisymmet- ric pieces, given by $Q^{S}(\psi, \theta)=[Q(\psi, \theta)+Q(\psi,-\theta)] / 2$ and $Q^{A}(\psi, \theta)=[Q(\psi, \theta)-Q(\psi,-\theta)] / 2$ for $Q$, and by $\bar{Q}^{S}(\psi, \theta)=[\bar{Q}(\psi, \theta)+\bar{Q}(\psi,-\theta)] / 2$ and $\bar{Q}^{A}(\psi, \theta)=$ $[\bar{Q}(\psi, \theta)-\bar{Q}(\psi,-\theta)] / 2$ for $\bar{Q}$. In a tokamak close to updown symmetry, there is a poloidal angle definition $\theta$ for which $Q^{A} \ll Q^{S}$ and $\bar{Q}^{S} \ll \bar{Q}^{A}$. In the gyrokinetic equation (19) and the Maxwell equations (28)-(30), the quantities $Q$ and $\bar{Q}$ enter in different coefficients. For small up-down symmetry, we can expand in $Q^{A} / Q^{S} \ll 1$ and $\bar{Q}^{S} / \bar{Q}^{A} \ll 1$, leaving to lowest order a system of gyrokinetic equations that only depend on $Q^{S}$ and $\bar{Q}^{A}$. In this case, the lowest order solutions $h_{s}^{(0)}, \phi^{(0)}, A_{\|}^{(0)}$ and $B_{\|}^{(0)}$ have the symmetry properties of the up-down symmetric case and the momentum flux is zero. For example,

$$
\begin{aligned}
\pi_{s, \|}^{\phi(0)} & =\frac{4 \pi^{2} i m_{s} c I}{V^{\prime}} \sum_{k_{\psi}, k_{\alpha}} k_{\alpha} \int d \theta \mathcal{J}^{S} \phi^{(0)}\left(k_{\psi}, k_{\alpha}\right) \\
& \times \int d w_{\|} d \mu h_{s}^{(0)}\left(-k_{\psi},-k_{\alpha}\right) w_{\|} J_{0}\left(z_{s}^{S}\right)=0,
\end{aligned}
$$

where by using the superscript $S$ we have emphasized that to this order $\mathcal{J}$ and $z_{s}$ are evaluated using only the up-down symmetric coefficients $Q^{S}$ and $\bar{Q}^{A}$. The next order correction to the system of gyrokinetic equations is linear in the next order corrections $h_{s}^{(1)}, \phi^{(1)}, A_{\|}^{(1)}$ and $B_{\|}^{(1)}$ to the lowest order solutions, and contains the antisymmetric coefficients $Q^{A}$ and $\bar{Q}^{S}$. As a result,

$$
\frac{h_{s}^{(1)}}{h_{s}^{(0)}} \sim \frac{\phi^{(1)}}{\phi^{(0)}} \sim \frac{A_{\|}^{(1)}}{A_{\|}^{(0)}} \sim \frac{B_{\|}^{(1)}}{B_{\|}^{(0)}} \sim \frac{Q^{A}}{Q^{S}} \sim \frac{\bar{Q}^{S}}{\bar{Q}^{A}} \ll 1 .
$$

Since the lowest order momentum flux is zero, the contribution due to these higher order correction becomes the only contribution. For example, to next order we find

$$
\begin{aligned}
\pi_{s, \|}^{\phi(1)}= & \frac{4 \pi^{2} i m_{s} c I}{V^{\prime}} \sum_{k_{\psi}, k_{\alpha}} k_{\alpha} \int d \theta \mathcal{J}^{S} \phi^{(1)}\left(k_{\psi}, k_{\alpha}\right) \\
& \times \int d w_{\|} d \mu h_{s}^{(0)}\left(-k_{\psi},-k_{\alpha}\right) w_{\|} J_{0}\left(z_{s}^{S}\right) \\
+ & \frac{4 \pi^{2} i m_{s} c I}{V^{\prime}} \sum_{k_{\psi}, k_{\alpha}} k_{\alpha} \int d \theta \mathcal{J}^{S} \phi^{(0)}\left(k_{\psi}, k_{\alpha}\right) \\
& \times \int d w_{\|} d \mu h_{s}^{(1)}\left(-k_{\psi},-k_{\alpha}\right) w_{\|} J_{0}\left(z_{s}^{S}\right) \\
+ & \frac{4 \pi^{2} i m_{s} c I}{V^{\prime}} \sum_{k_{\psi}, k_{\alpha}} k_{\alpha} \int d \theta \mathcal{J}^{A} \phi^{(0)}\left(k_{\psi}, k_{\alpha}\right) \\
& \times \int d w_{\|} d \mu h_{s}^{(0)}\left(-k_{\psi},-k_{\alpha}\right) w_{\|} J_{0}\left(z_{s}^{S}\right) \\
- & \frac{4 \pi^{2} i m_{s} c I}{V^{\prime}} \sum_{k_{\psi}, k_{\alpha}} k_{\alpha} \int d \theta \mathcal{J}^{S} \phi^{(0)}\left(k_{\psi}, k_{\alpha}\right) \\
& \times \int d w_{\|} d \mu h_{s}^{(0)}\left(-k_{\psi},-k_{\alpha}\right) w_{\|} J_{1}\left(z_{s}^{S}\right) z_{s}^{A},
\end{aligned}
$$


where the superscript $A$ in $\mathcal{J}^{A}$ and $z_{s}^{A}$ indicates that these are the next order corrections in $Q^{A} / Q^{S} \ll 1$ and $\bar{Q}^{S} / \bar{Q}^{A} \ll 1$ to the coefficients $\mathcal{J}$ and $z_{s}$, and as a consequence, they contain $Q^{A}$ and $\bar{Q}^{S}$. Note that $\pi_{s, \|}^{\phi(1)}$ contains terms that have exactly the opposite symmetry to the symmetry that made the lowest order contribution $\pi_{s, \|}^{\phi(0)}$ zero. Thus, the contribution to momentum flux due to up-down asymmetry is proportional to the asymmetric component of the equilibrium coefficients, that is, proportional to $Q^{A}$ and $\bar{Q}^{S}$.

To demonstrate that the symmetry based on transformation I is observed in local $\delta f$ gyrokinetic simulations, the local $\delta f$ gyrokinetic code GS2 [3] was used to solve the gyrokinetic system of equations $(19)$ and $(28)$ for an updown symmetric equilibrium with $\Omega_{\zeta}=0$, but with three different values of $\partial \Omega_{\zeta} / \partial \psi$ : zero and $\pm 0.2 q^{2} v_{t i} / \varepsilon^{2} R_{0}^{3} B_{0}$. Here $v_{t i}=\sqrt{T_{i} / m_{i}}, T_{i}$ and $m_{i}$ are the ion thermal speed, temperature and mass, $R_{0}$ and $B_{0}$ are the major radius and the magnetic field at the magnetic axis, $q=1.4$ is the safety factor, and $\varepsilon=0.18$ is the inverse aspect ratio. All other equilibrium parameters correspond to the Cyclone base case [31. Only electrostatic fluctuations are considered and a modified Boltzmann response 32 is employed for the electrons. From the solutions for $h_{i}$, we calculate the dimensionless function $\mathrm{P}\left(\mathrm{k}_{\psi}, \mathrm{k}_{\alpha}, \theta, \mathrm{w}_{\|}\right)$, defined as

$$
\begin{array}{r}
\mathrm{P}=\frac{2 \pi c k_{\alpha} \phi\left(k_{\psi}, k_{\alpha}\right)}{\epsilon_{i}^{2} R_{0} n_{i} v_{t i}\langle|\nabla \psi|\rangle_{\psi}} \int d \mu h_{i}\left(-k_{\psi},-k_{\alpha}\right) \\
\quad \times\left(i I w_{\|} J_{0}\left(z_{i}\right)-\frac{m_{i} c}{e} k^{\psi} \mu \frac{2 J_{1}\left(z_{i}\right)}{z_{i}}\right),
\end{array}
$$

where $n_{i}, p_{i}=n_{i} T_{i}$ and $\rho_{i}=m_{i} c v_{t i} / e B$ are ion density, pressure and thermal gyroradius, and $\epsilon_{i}=\rho_{i} / R_{0}$. The function $\mathrm{P}$ can be understood as the dimensionless contribution to the radial momentum flux due to fluctuations at the poloidal angle $\theta$ with dimensionless wavelength $\mathrm{k}_{\psi}=k_{\psi} \epsilon_{i}\left(\varepsilon R_{0}^{2} B_{0} / q\right)$ and $\mathrm{k}_{\alpha}=k_{\alpha} \epsilon_{i}(q / \varepsilon)$ when interacting with particles with dimensionless parallel velocity $\mathbf{w}_{\|}=w_{\|} / v_{t i}$. The function $\mathbf{P}$ is related to the turbulent toroidal angular momentum flux by

$$
\frac{\left\langle\pi_{i, \|}^{\phi}+\pi_{i, \perp}^{\phi}\right\rangle_{\Delta t}}{\epsilon_{i}^{2} R_{0} p_{i}\langle|\nabla \psi|\rangle_{\psi}}=\frac{2 \pi}{V^{\prime}} \sum_{\mathbf{k}_{\psi}, \mathrm{k}_{\alpha}} \int d \theta \mathcal{J} \int d \mathrm{w}_{\|} \mathrm{P} .
$$

From the symmetry under transformation $\mathrm{I}$ in Table [I] we find that $\left\langle\mathrm{P}\left(\mathrm{k}_{\psi}, \mathrm{k}_{\alpha}, \mathrm{w}_{\|}, \theta\right)\right\rangle_{\Delta t}=$ $-\left\langle\mathrm{P}\left(-\mathrm{k}_{\psi}, \mathrm{k}_{\alpha},-\mathrm{w}_{\|},-\theta\right)\right\rangle_{\Delta t}$ for the case with $\partial \Omega_{\zeta} / \partial \psi=0$. The change in sign in the angle $\theta$ is due to the up-down reflection. As proven in Appendix F for every angle $\theta$ defined for an equilibrium, there is an angle $\theta$ for the up-down reflection such that Table 1 is satisfied. In this case, for which the up-down reflection is the flux surface itself, we choose the poloidal angle definition $\theta$ so that the corresponding poloidal angle for the up-down reflection is simply $-\theta$. The result
$\left\langle\mathrm{P}\left(\mathrm{k}_{\psi}, \mathrm{k}_{\alpha}, \mathrm{w}_{\|}, \theta\right)\right\rangle_{\Delta t}=-\left\langle\mathrm{P}\left(-\mathrm{k}_{\psi}, \mathrm{k}_{\alpha},-\mathrm{w}_{\|},-\theta\right)\right\rangle_{\Delta t}$ is confirmed by Figs. 2(a) and 2(b), which clearly show that the integrals of the function $\mathrm{P}\left(\mathrm{k}_{\psi}, \mathrm{k}_{\alpha}, \theta, \mathrm{w}_{\|}\right)$

$$
\mathrm{P}_{\mathrm{w}_{\|}, \theta}\left(\mathrm{w}_{\|}, \theta\right)=\left\langle\sum_{\mathrm{k}_{\psi}, \mathrm{k}_{\alpha}} \mathrm{P}\right\rangle_{\Delta t}
$$

and

$$
\mathrm{P}_{\mathrm{k}_{\psi}, \mathrm{k}_{\alpha}}\left(\mathrm{k}_{\psi}, \mathrm{k}_{\alpha}\right)=\left\langle\frac{2 \pi}{V^{\prime}} \int d \theta \mathcal{J} \int d \mathrm{w}_{\|} \mathrm{P}\right\rangle_{\Delta t}
$$

satisfy the relations

$$
\mathrm{P}_{\mathrm{w}_{\|}, \theta}\left(\mathrm{w}_{\|}, \theta\right)=-\mathrm{P}_{\mathrm{w}_{\|}, \theta}\left(-\mathrm{w}_{\|},-\theta\right)
$$

and

$$
\mathrm{P}_{\mathrm{k}_{\psi}, \mathrm{k}_{\alpha}}\left(\mathrm{k}_{\psi}, \mathrm{k}_{\alpha}\right)=-\mathrm{P}_{\mathrm{k}_{\psi}, \mathrm{k}_{\alpha}}\left(-\mathrm{k}_{\psi}, \mathrm{k}_{\alpha}\right) .
$$

This symmetry is broken for the cases with finite $\partial \Omega_{\zeta} / \partial \psi$, which are shown in Figs. 2(c)-2(f). Observe that the symmetry also gives that the case with negative $\partial \Omega_{\zeta} / \partial \psi$ has $\mathrm{P}_{-}\left(\mathrm{k}_{\psi}, \mathrm{k}_{\alpha}, \mathrm{w}_{\|}, \theta\right)=$ $-\mathrm{P}_{+}\left(-\mathrm{k}_{\psi}, \mathrm{k}_{\alpha},-\mathrm{w}_{\|},-\theta\right)$, where $\mathrm{P}_{+}\left(\mathrm{k}_{\psi}, \mathrm{k}_{\alpha}, \mathrm{w}_{\|}, \theta\right)$ is the function $\mathrm{P}$ for the case with positive $\partial \Omega_{\zeta} / \partial \psi$, and $\mathrm{P}_{-}\left(\mathrm{k}_{\psi}, \mathrm{k}_{\alpha}, \mathrm{w}_{\|}, \theta\right)$ is the function $\mathrm{P}$ for the negative $\partial \Omega_{\zeta} / \partial \psi$. This is confirmed by comparing Fig. 2(c) with 2(e), and Fig. 2(d) with 2(f). As a consequence of these symmetries, the case with $\partial \Omega_{\zeta} / \partial \psi=0$ has $\left\langle\pi_{i, \|}^{\phi}+\pi_{i, \perp}^{\phi}\right\rangle_{\Delta t}=0$, while the other cases have $\left\langle\pi_{i, \|}^{\phi}+\right.$ $\left.\pi_{i, \perp}^{\phi}\right\rangle_{\Delta t}= \pm 2.6 \epsilon_{i}^{2} R_{0} p_{i}\langle|\nabla \psi|\rangle_{\psi}$.

Following the same reasoning as for transformation I, transformation II in Table II implies that the transport of momentum remains the same for an up-down reflection and a reversal of the toroidal magnetic field $B_{\zeta}=I / R$. This means then that for an up-down symmetric tokamak, the transport of momentum cannot depend on the direction of the toroidal magnetic field. For example, the Coriolis pinch 7 will remain the same under a reversal of the toroidal magnetic field.

These symmetries of the lowest order gyrokinetic formulation have very important consequences for the modeling of spontaneous rotation profiles in tokamaks without net momentum input. When no momentum is injected, the radial momentum flux across any flux surface within the separatrix must vanish, i.e., $\Pi(\psi)=0$. In the absence of up-down asymmetry, the radial transport of momentum given by the lowest order gyrokinetic model vanishes for $\Omega_{\zeta}=0$ and $\partial \Omega_{\zeta} / \partial \psi=0$. Thus, for zero or negligible velocity in the edge, the solution to the equation $\Pi\left(\Omega_{\zeta}, \partial \Omega_{\zeta} / \partial \psi\right)=0$ is $\Omega_{\zeta}(\psi)=0$ everywhere. To obtain $\Omega_{\zeta}(\psi) \neq 0$ with the lowest order gyrokinetic formulation, we need to assume that there is some up-down asymmetry $[9$. This limits the applicability of the lowest order formulation to studies of spontaneous rotation in very up-down asymmetric configurations. When higher order terms are considered [17, 18, more mechanisms for the generation of spontaneous rotation arise. 
(a)

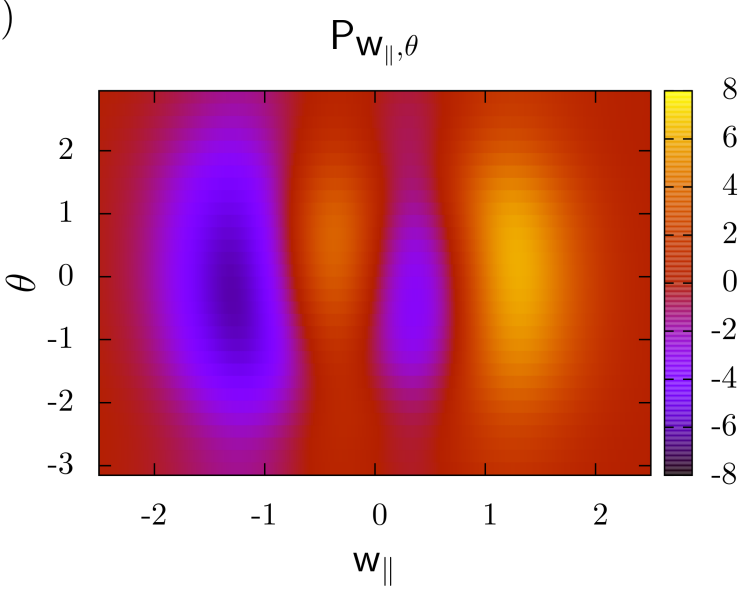

(c)

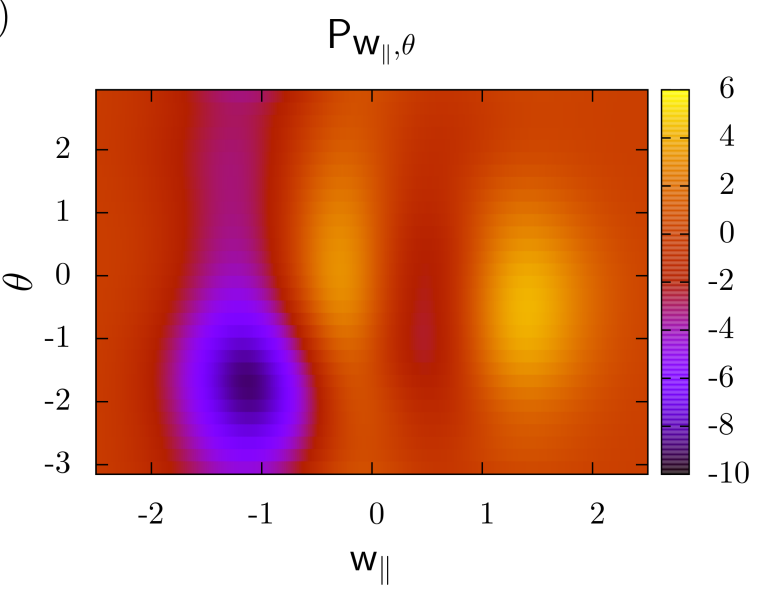

(e)

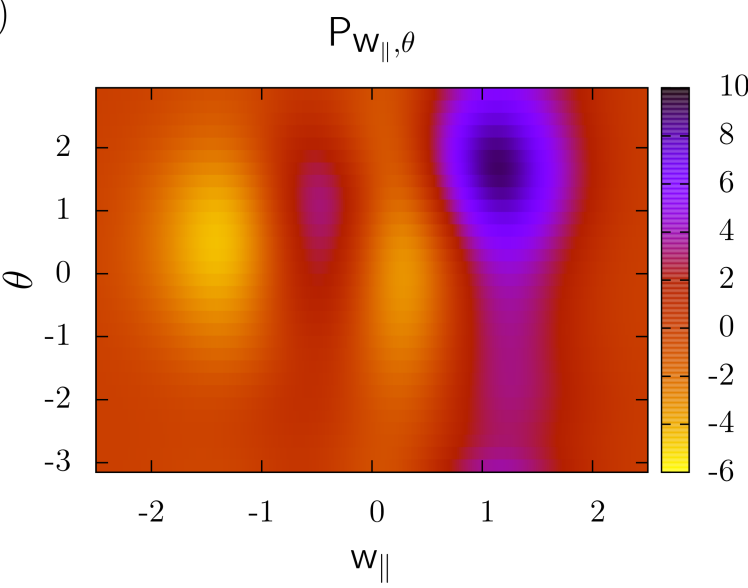

(b)

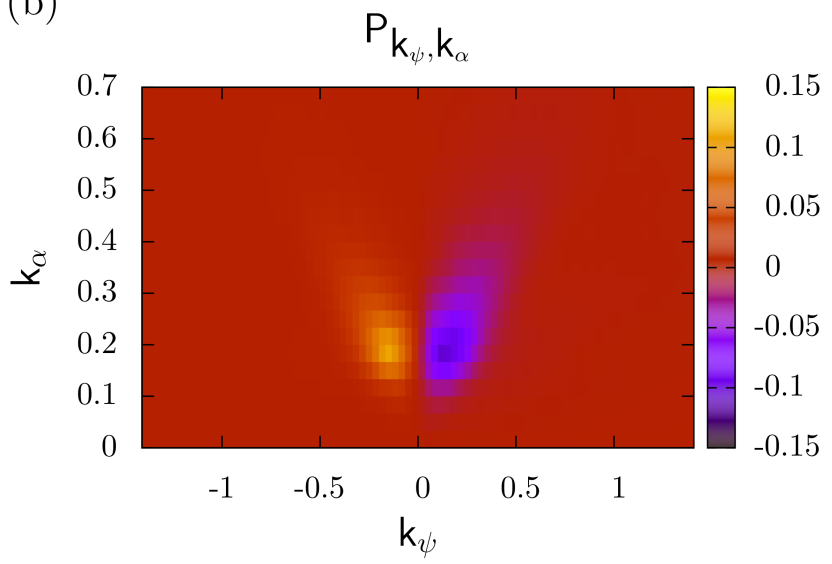

(d)

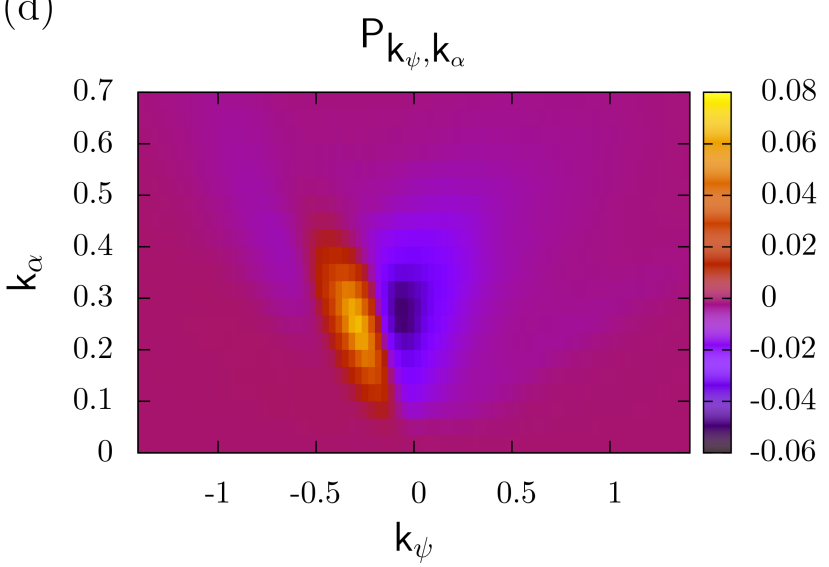

(f)

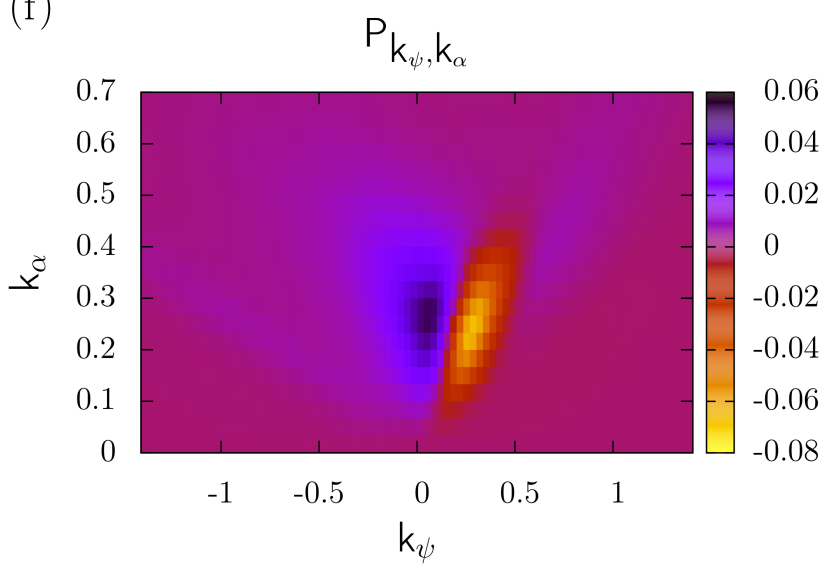

FIG. 2: (Color online) Contributions to momentum transport for different values of $\partial \Omega_{\zeta} / \partial \psi$ : (a)-(b) $\partial \Omega_{\zeta} / \partial \psi=0$; (c)-(d) $\partial \Omega_{\zeta} / \partial \psi=0.2 q^{2} v_{t i} / \varepsilon^{2} R_{0}^{3} B_{0}$; and (e)-(f) $\partial \Omega_{\zeta} / \partial \psi=-0.2 q^{2} v_{t i} / \varepsilon^{2} R_{0}^{3} B_{0}$. Note that in figures (e) and (f) the color map has been inverted to aid the comparison with figures (c) and (d).

\section{Acknowledgments}

The authors are grateful for the hospitality of the Isaac Newton Institute for Mathematical Sciences at University of Cambridge. This article is the final result of a series of conversations at the summer programme "Gyroki- netics in Laboratory and Astrophysical Plasmas" hosted there.

This work was supported in part by the postdoctoral fellowship programme of UK EPSRC, the JRF programme of Christ Church at University of Oxford, the Oxford-Culham Fusion Research Fellowship and the Lev- 
erhulme Trust Network for Magnetized Plasma Turbulence.

\section{Appendix A: Collision operator}

The linearized collision operator is given by

$$
\bar{C}_{s s^{\prime}}^{(\ell)}=\nabla_{w} \cdot\left(\overline{\boldsymbol{\Gamma}}_{s s^{\prime}}^{\lambda}+\overline{\boldsymbol{\Gamma}}_{s s^{\prime}}^{\varepsilon}+\overline{\boldsymbol{\Gamma}}_{s s^{\prime}}^{R}\right) .
$$

The different contributions to the collision operator are the pitch-angle scattering

$$
\overline{\mathbf{\Gamma}}_{s s^{\prime}}^{\lambda}=\nu_{s s^{\prime}}^{\lambda}\left(w^{2} \stackrel{\leftrightarrow}{\mathbf{I}}-\mathbf{w w}\right) \cdot \nabla_{w} \bar{h}_{s}
$$

the energy scattering

$$
\overline{\boldsymbol{\Gamma}}_{s s^{\prime}}^{\varepsilon}=\nu_{s s^{\prime}}^{\varepsilon} \mathbf{w}\left(\mathbf{w} \cdot \nabla_{w} \bar{h}_{s}+\frac{m_{s} w^{2}}{T_{s^{\prime}}} \bar{h}_{s}\right),
$$

and the integral contribution

$$
\overline{\boldsymbol{\Gamma}}_{s s^{\prime}}^{R}=-\frac{m_{s} \gamma_{s s^{\prime}} f_{M s}}{T_{s}}\left(\mathbf{w} \cdot \nabla_{w} \nabla_{w} \bar{H}_{s^{\prime}}+\frac{T_{s}}{m_{s^{\prime}}} \nabla_{w} \bar{L}_{s^{\prime}}\right)
$$

where the Rosenbluth potentials $\bar{H}_{s^{\prime}}$ and $\bar{L}_{s^{\prime}}$ are obtained by solving the differential equations $\nabla_{w}^{2} \bar{L}_{s^{\prime}}=-8 \pi \bar{h}_{s^{\prime}}$ and $\nabla_{w}^{2} \bar{H}_{s^{\prime}}=\bar{L}_{s^{\prime}}$. Here $\gamma_{s s^{\prime}}=2 \pi Z_{s}^{2} Z_{s^{\prime}}^{2} e^{4} \ln \Lambda / m_{s}^{2}, \ln \Lambda$ is Coulomb's logarithm,

$$
\nu_{s s^{\prime}}^{\lambda}(w)=\frac{\gamma_{s s^{\prime}} n_{s^{\prime}} m_{s^{\prime}}^{3 / 2}}{2^{3 / 2} T_{s^{\prime}}^{3 / 2}}\left(\frac{2 \bar{w}_{s^{\prime}}^{2}-1}{2 \bar{w}_{s^{\prime}}^{5}} \Phi\left(\bar{w}_{s^{\prime}}\right)+\frac{1}{2 \bar{w}_{s^{\prime}}^{4}} \frac{d \Phi}{d \bar{w}_{s^{\prime}}}\right)
$$

is the pitch-angle scattering collision frequency, and

$$
\nu_{s s^{\prime}}^{\varepsilon}(w)=\frac{\gamma_{s s^{\prime}} n_{s^{\prime}} m_{s^{\prime}}^{3 / 2}}{2^{3 / 2} T_{s^{\prime}}^{3 / 2}}\left(\frac{\Phi\left(\bar{w}_{s^{\prime}}\right)}{\bar{w}_{s^{\prime}}^{5}}-\frac{1}{\bar{w}_{s^{\prime}}^{4}} \frac{d \Phi}{d \bar{w}_{s^{\prime}}}\right)
$$

is the energy scattering collision frequency. We have used the abbreviated notation $\bar{w}_{s^{\prime}}=w / \sqrt{2 T_{s^{\prime}} / m_{s^{\prime}}}$, $\Phi\left(\bar{w}_{s^{\prime}}\right)=(2 / \sqrt{\pi}) \int_{0}^{\bar{w}_{s^{\prime}}} d y \exp \left(-y^{2}\right)$ and $d \Phi / d \bar{w}_{s^{\prime}}=$ $(2 / \sqrt{\pi}) \exp \left(-\bar{w}_{s^{\prime}}^{2}\right)$.

\section{Appendix B: Derivation of the local $\delta f$ kinetic equation (19)}

In this Appendix we derive 19 from (8). We Fourier analyze the equation term by term.

We change from the spatial coordinates $\mathbf{X}$ to the new coordinates $\psi(\mathbf{X}), \alpha(\mathbf{X}, t)$ and $\theta(\mathbf{X})$. After this coordinate transformation, and after Fourier transforming, the term $\partial \bar{h}_{s} / \partial t+R \Omega_{\zeta} \hat{\boldsymbol{\zeta}} \cdot \nabla_{\mathbf{X}} \bar{h}_{s}$ becomes $\partial h_{s} / \partial t+$ $\left[(\partial \alpha / \partial t)+R \Omega_{\zeta} \hat{\boldsymbol{\zeta}} \cdot \nabla \alpha\right] i k_{\alpha} h_{s}$. We drop the subscript $\mathbf{x}$ in $\nabla_{\mathbf{X}}$ for simplicity. Employing $\partial \alpha / \partial t+R \Omega_{\zeta} \hat{\boldsymbol{\zeta}} \cdot \nabla \alpha=$ $0, \partial \bar{h}_{s} / \partial t+R \Omega_{\zeta} \hat{\boldsymbol{\zeta}} \cdot \nabla_{\mathbf{X}} \bar{h}_{s}$ becomes $\partial h_{s} / \partial t$. Similarly, the Fourier transform of $\partial\langle\bar{\chi}\rangle_{s} / \partial t+R \Omega_{\zeta} \hat{\boldsymbol{\zeta}} \cdot \nabla_{\mathbf{X}}\langle\bar{\chi}\rangle_{s}$ is $\partial\langle\chi\rangle_{s} / \partial t$.
The Fourier transform of $\mathbf{v}_{d, s} \cdot \nabla_{\mathbf{X}} \bar{h}_{s}$ is $i k_{\psi} h_{s} \mathbf{v}_{d, s} \cdot \nabla \psi+$ $i k_{\alpha} h_{s} \mathbf{v}_{d, s} \cdot \nabla \alpha+\mathbf{v}_{d, s} \cdot \nabla \theta\left(\partial h_{s} / \partial \theta\right)$. The contribution $\mathbf{v}_{d, s} \cdot \nabla \theta(\partial / \partial \theta)$ is negligible since we assume that the parallel derivative $\partial / \partial \theta$ is small compared to $k_{\perp}$. Before evaluating $\mathbf{v}_{d, s} \cdot \nabla \psi$ and $\mathbf{v}_{d, s} \cdot \nabla \alpha$, we rewrite the curvature drift by employing the MHD equilibrium result $\hat{\mathbf{b}} \cdot \nabla \hat{\mathbf{b}}=B^{-1} \nabla_{\perp} B+\left(4 \pi / B^{2}\right)\left(\partial p /\left.\partial \psi\right|_{R}\right) \nabla \psi$. Then, defining $v_{d, s}^{\psi}=\mathbf{v}_{d, s} \cdot \nabla \psi$ and $v_{d, s}^{\alpha}=\mathbf{v}_{d, s} \cdot \nabla \alpha$, and employing that the gradient of the axisymmetric quantities $\Phi_{0}, B$ and $R$ can be written as $\nabla_{\mathbf{X}}=\nabla \psi(\partial / \partial \psi)+\nabla \theta(\partial / \partial \theta)$, we find expressions $(25)$ and $(26)$. To obtain the final form of these equations we have used $(\nabla \theta \times \hat{\boldsymbol{\zeta}}) \cdot \nabla \psi=R \mathbf{B} \cdot \nabla \theta$ for the $\psi$ component of $\mathbf{v}_{c o, s}, \hat{\mathbf{b}} \times \nabla \psi=I \hat{\mathbf{b}}-R B \hat{\boldsymbol{\zeta}}$ for the $\psi$ component of $\mathbf{v}_{E 0}, \mathbf{v}_{M, s}$ and $\mathbf{v}_{c f, s}$, and $(\nabla \alpha \times \nabla \psi) \cdot \hat{\mathbf{b}}=B$ for the $\alpha$ component of $\mathbf{v}_{E 0}, \mathbf{v}_{M, s}$ and $\mathbf{v}_{c f, s}$.

The nonlinear term $\left\{\langle\chi\rangle_{s}, h_{s}\right\}$ is the Fourier transform of $\overline{\mathbf{v}}_{\chi, s} \cdot \nabla_{\mathbf{X}} \bar{h}_{s}$. The generalized potential $\langle\bar{\chi}\rangle_{s}$ in 10$\rangle$ can be Fourier transformed by realizing that

$$
\langle\bar{\chi}\rangle_{s}=\sum_{k_{\psi}, k_{\alpha}}\langle\chi \exp (i S(\mathbf{x}))\rangle_{s}
$$

where $\chi=\phi-c^{-1}\left(w_{\|} A_{\|}+\mathbf{w}_{\perp} \cdot \mathbf{A}_{\perp}\right)$ and $S(\mathbf{x})=k_{\psi} \psi(\mathbf{x})+$ $k_{\alpha} \alpha(\mathbf{x})$. Using $S(\mathbf{x})=S\left(\mathbf{X}+\boldsymbol{\rho}_{s}\right) \simeq S(\mathbf{X})+\mathbf{k}_{\perp} \cdot \boldsymbol{\rho}_{s}$, with $\mathbf{k}_{\perp}=k_{\psi} \nabla \psi+k_{\alpha} \nabla \alpha$, we find that the Fourier transform of B1 is

$$
\begin{array}{r}
\langle\chi\rangle_{s}=\frac{1}{2 \pi}\left(\phi-\frac{1}{c} w_{\|} A_{\|}\right) \int d \varphi \exp \left(i \mathbf{k}_{\perp} \cdot \boldsymbol{\rho}_{s}\right) \\
-\frac{1}{2 \pi c} \mathbf{A}_{\perp} \cdot \int d \varphi \mathbf{w}_{\perp} \exp \left(i \mathbf{k}_{\perp} \cdot \boldsymbol{\rho}_{s}\right) .
\end{array}
$$

To obtain the final result in 20 , we need to employ $B_{\|}=i \hat{\mathbf{b}} \cdot\left(\mathbf{k}_{\perp} \times \mathbf{A}_{\perp}\right)$,

$$
\int d \varphi \exp \left(i \mathbf{k}_{\perp} \cdot \boldsymbol{\rho}_{s}\right)=2 \pi J_{0}\left(z_{s}\right)
$$

and

$$
\int d \varphi \mathbf{w}_{\perp} \exp \left(i \mathbf{k}_{\perp} \cdot \boldsymbol{\rho}_{s}\right)=-2 \pi \frac{i m_{s} c \mu}{Z_{s} e} \frac{2 J_{1}\left(z_{s}\right)}{z_{s}} \hat{\mathbf{b}} \times \mathbf{k}_{\perp},
$$

where $z_{s}$ is defined in 21 . To prove $(\mathrm{B} 3$, we use (11) to write $\mathbf{k}_{\perp} \cdot \boldsymbol{\rho}_{s}=-\left(\sqrt{2 \mu B} / \Omega_{s}|\nabla \psi|\right)\left[\left(k_{\psi}|\nabla \psi|^{2}+k_{\alpha} \nabla \alpha\right.\right.$. $\left.\nabla \psi) \sin \varphi+B k_{\alpha} \cos \varphi\right]$. Then,

$$
\mathbf{k}_{\perp} \cdot \boldsymbol{\rho}_{s}=-z_{s} \sin \left(\varphi+\varphi_{\mathbf{k}}\right)
$$

with $\varphi_{\mathbf{k}}=\arctan \left[B k_{\alpha} /\left(k_{\psi}|\nabla \psi|^{2}+k_{\alpha} \nabla \alpha \cdot \nabla \psi\right)\right]$. Using this expression and

$$
\exp (i z \sin \varphi)=\sum_{n=-\infty}^{\infty} J_{n}(z) \exp (i n \varphi)
$$

we obtain (B3). Equation (B4) is obtained by realizing that $\mathbf{w}_{\perp} \exp \left(i \mathbf{k}_{\perp} \cdot \boldsymbol{\rho}_{s}\right)=i \Omega_{s} \mathbf{b} \times \nabla_{\mathbf{k}_{\perp}} \exp \left(i \mathbf{k}_{\perp} \cdot \boldsymbol{\rho}_{s}\right)$ and hence $\int d \varphi \mathbf{w}_{\perp} \exp \left(i \mathbf{k}_{\perp} \cdot \boldsymbol{\rho}_{s}\right)=2 \pi i \Omega_{s} \hat{\mathbf{b}} \times \nabla_{\mathbf{k}_{\perp}} J_{0}\left(z_{s}\right)$. 
Finally, the last term in the right side of 19 is the Fourier transform of the last term in (8). To obtain the final result in $(19)$, we use $(3)$ to find

$$
\frac{1}{n_{s}} \frac{\partial n_{s}}{\partial \theta}+\frac{Z_{s} e}{T_{s}} \frac{\partial \Phi_{0}}{\partial \theta}-\frac{m_{s} R \Omega_{\zeta}^{2}}{T_{s}} \frac{\partial R}{\partial \theta}=0
$$

\section{Appendix C: Gyroaveraged collision operator}

In this Appendix we Fourier transform the gyroaverage of the collision operator given in Appendix A. To obtain the Fourier transform, we need to express the collision operator as

$$
\left\langle\bar{C}_{s s^{\prime}}^{(\ell)}\right\rangle_{s}=\sum_{k_{\psi}, k_{\alpha}}\left\langle C_{s s^{\prime}}^{(\ell)}\right\rangle_{s} \exp (i S(\mathbf{X}))
$$

where $S(\mathbf{X})=k_{\psi} \psi(\mathbf{X})+k_{\alpha} \alpha(\mathbf{X})$ depends on the gyrocenter position $\mathbf{X}$. This Appendix contains the procedures that we use to write the different terms in the gyroaveraged collision operator as in (C1). Once the operator is expressed in this way, it is easy to see that the coefficients $\left\langle C_{s s^{\prime}}^{(\ell)}\right\rangle_{s}$ are the ones that appear in 19 .

To differentiate with respect to $\mathbf{w}$ in $\nabla_{w} \cdot \Gamma_{s s^{\prime}}^{\lambda}$ and $\nabla_{w} \cdot \overline{\boldsymbol{\Gamma}}_{s s^{\prime}}^{\varepsilon}$, we use that

$$
\bar{h}_{s}=\sum_{k_{\psi}, k_{\alpha}} h_{s} \exp (i S(\mathbf{X}))
$$

Employing $\nabla_{w} w_{\|}=\hat{\mathbf{b}}, \nabla_{w} \mu=\mathbf{w}_{\perp} / B$ and $\nabla_{w} S(\mathbf{X})=$ $\nabla_{w} \mathbf{X} \cdot \mathbf{k}_{\perp}=\Omega_{s}^{-1} \hat{\mathbf{b}} \times \mathbf{k}_{\perp}$, we find that the Fourier transforms of the gyroaveraged divergences of the vectors $\overline{\boldsymbol{\Gamma}}_{s s^{\prime}}^{\lambda}$ and $\overline{\boldsymbol{\Gamma}}_{s s^{\prime}}^{\varepsilon}$ are 33 .

$$
\left\langle\nabla_{w} \cdot \Gamma_{s s^{\prime}}^{\lambda}\right\rangle_{s}=\frac{\partial \Gamma_{s s^{\prime}, w_{\|}}^{\lambda}}{\partial w_{\|}}+\frac{\partial \Gamma_{s s^{\prime}, \mu}^{\lambda}}{\partial \mu}-\frac{k_{\perp}^{2} \mu B}{\Omega_{s}^{2}} D_{s s^{\prime}}^{\lambda}
$$

and

$$
\left\langle\nabla_{w} \cdot \Gamma_{s s^{\prime}}^{\varepsilon}\right\rangle_{s}=\frac{\partial \Gamma_{s s^{\prime}, w_{\|}}^{\varepsilon}}{\partial w_{\|}}+\frac{\partial \Gamma_{s s^{\prime}, \mu}^{\varepsilon}}{\partial \mu}-\frac{k_{\perp}^{2} \mu B}{\Omega_{s}^{2}} D_{s s^{\prime}}^{\varepsilon} .
$$

Here the pitch-angle scattering terms are

$$
\begin{gathered}
\Gamma_{s s^{\prime}, w_{\|}}^{\lambda}=2 \mu \nu_{s s^{\prime}}^{\lambda}\left(B \frac{\partial h_{s}}{\partial w_{\|}}-w_{\|} \frac{\partial h_{s}}{\partial \mu}\right), \\
\Gamma_{s s^{\prime}, \mu}^{\lambda}=2 \mu \nu_{s s^{\prime}}^{\lambda}\left(-w_{\|} \frac{\partial h_{s}}{\partial w_{\|}}+\frac{w_{\|}^{2}}{B} \frac{\partial h_{s}}{\partial \mu}\right)
\end{gathered}
$$

and $D_{s s^{\prime}}^{\lambda}=\left[1+\left(w_{\|}^{2} / \mu B\right)\right] \nu_{s s^{\prime}}^{\lambda} h_{s}$, and the energy scattering terms are

$$
\Gamma_{s s^{\prime}, w_{\|}}^{\varepsilon}=w_{\|} \nu_{s s^{\prime}}^{\varepsilon}\left(w_{\|} \frac{\partial h_{s}}{\partial w_{\|}}+2 \mu \frac{\partial h_{s}}{\partial \mu}+\frac{m_{s} w^{2}}{T_{s^{\prime}}} h_{s}\right),
$$

$$
\Gamma_{s s^{\prime}, \mu}^{\varepsilon}=2 \mu \nu_{s s^{\prime}}^{\varepsilon}\left(w_{\|} \frac{\partial h_{s}}{\partial w_{\|}}+2 \mu \frac{\partial h_{s}}{\partial \mu}+\frac{m_{s} w^{2}}{T_{s^{\prime}}} h_{s}\right)
$$

and $D_{s s^{\prime}}^{\varepsilon}=\nu_{s s^{\prime}}^{\varepsilon} h_{s}$. Note that $w^{2}=w_{\|}^{2}+2 \mu B$.

To Fourier transform the gyroaveraged divergence of $\overline{\boldsymbol{\Gamma}}_{s s^{\prime}}^{R}$, it is convenient to write the Rosenbluth potentials $\bar{H}_{s^{\prime}}\left(\mathbf{x}, w_{\|}, \mu, \varphi, t\right)$ and $\bar{L}_{s^{\prime}}\left(\mathbf{x}, w_{\|}, \mu, \varphi, t\right)$ as functions of the position of the particle $\mathbf{x}$ instead of the guiding center $\mathbf{X}$. To obtain $\bar{H}_{s^{\prime}}$ and $\bar{L}_{s^{\prime}}$ from equations $\nabla_{w}^{2} \bar{L}_{s^{\prime}}=-8 \pi \bar{h}_{s^{\prime}}$ and $\nabla_{w}^{2} \bar{H}_{s^{\prime}}=\bar{L}_{s^{\prime}}$, we use C2, where $S(\mathbf{X})=S\left(\mathbf{x}-\boldsymbol{\rho}_{s}\right) \simeq S(\mathbf{x})-\mathbf{k}_{\perp} \cdot \boldsymbol{\rho}_{s}$, and we employ B5 and (B6) to write

$$
\bar{h}_{s}=\sum_{k_{\psi}, k_{\alpha}} \sum_{n=-\infty}^{\infty} J_{n}\left(z_{s}\right) h_{s} \exp \left(i n\left(\varphi+\varphi_{\mathbf{k}}\right)+i S(\mathbf{x})\right) .
$$

Using the decompositions

$$
\bar{L}_{s^{\prime}}=\sum_{k_{\psi}, k_{\alpha}} \sum_{n=-\infty}^{\infty} L_{s^{\prime}, n} \exp \left(i n\left(\varphi+\varphi_{\mathbf{k}}\right)+i S(\mathbf{x})\right)
$$

and

$$
\bar{H}_{s^{\prime}}=\sum_{k_{\psi}, k_{\alpha}} \sum_{n=-\infty}^{\infty} H_{s^{\prime}, n} \exp \left(i n\left(\varphi+\varphi_{\mathbf{k}}\right)+i S(\mathbf{x})\right),
$$

we obtain the functions $L_{s^{\prime}, n}\left(k_{\psi}, k_{\alpha}, w_{\|}, \mu, t\right)$ and $H_{s^{\prime}, n}\left(k_{\psi}, k_{\alpha}, w_{\|}, \mu, t\right)$ from equations

$$
\frac{\partial^{2} L_{s^{\prime}, n}}{\partial w_{\|}^{2}}+\frac{2}{B} \frac{\partial}{\partial \mu}\left(\mu \frac{\partial L_{s^{\prime}, n}}{\partial \mu}\right)-\frac{n^{2}}{2 \mu B} L_{s^{\prime}, n}=-8 \pi J_{n}\left(z_{s^{\prime}}\right) h_{s^{\prime}}
$$

and

$$
\frac{\partial^{2} H_{s^{\prime}, n}}{\partial w_{\|}^{2}}+\frac{2}{B} \frac{\partial}{\partial \mu}\left(\mu \frac{\partial H_{s^{\prime}, n}}{\partial \mu}\right)-\frac{n^{2}}{2 \mu B} H_{s^{\prime}, n}=L_{s^{\prime}, n} .
$$

With the decompositions C10 and C11, and $\nabla_{w} w_{\|}=$ $\hat{\mathbf{b}}, \nabla_{w} \mu=\mathbf{w}_{\perp} / B, \nabla_{w} \varphi=-\mathbf{w} \times \hat{\mathbf{b}} / 2 \mu B, \nabla_{w} \nabla_{w} w_{\|}=0$, $\nabla_{w} \nabla_{w} \mu=(\overleftrightarrow{\mathbf{I}}-\hat{\mathbf{b}} \hat{\mathbf{b}}) / B$ and $\nabla_{w} \nabla_{w} \varphi=\left[\mathbf{w}_{\perp}(\mathbf{w} \times \hat{\mathbf{b}})+\right.$ $\left.(\mathbf{w} \times \hat{\mathbf{b}}) \mathbf{w}_{\perp}\right] / 4 \mu^{2} B^{2}$, we obtain

$$
\begin{array}{r}
\nabla_{w} \cdot \overline{\boldsymbol{\Gamma}}_{s s^{\prime}}^{R}=\sum_{k_{\psi}, k_{\alpha}} \sum_{n=-\infty}^{\infty}\left[\frac{\partial G_{s s^{\prime}, w_{\|}, n}^{R}}{\partial w_{\|}}+\frac{\partial G_{s s^{\prime}, \mu, n}^{R}}{\partial \mu}\right. \\
\left.-\frac{k_{\perp}^{2} \mu B}{\Omega_{s}^{2}} \Delta_{s s^{\prime}, n}^{R}\right] \exp \left(i n\left(\varphi+\varphi_{\mathbf{k}}\right)+i S(\mathbf{x})\right),(
\end{array}
$$

where

$$
\begin{aligned}
G_{s s^{\prime}, w_{\|}, n}^{R} & =-\frac{m_{s} \gamma_{s s^{\prime}} f_{M s}}{T_{s}}\left(w_{\|} \frac{\partial^{2} H_{s^{\prime}, n}}{\partial w_{\|}^{2}}\right. \\
& \left.+2 \mu \frac{\partial^{2} H_{s^{\prime}, n}}{\partial w_{\|} \partial \mu}+\frac{T_{s}}{m_{s^{\prime}}} \frac{\partial L_{s^{\prime}, n}}{\partial w_{\|}}\right)
\end{aligned}
$$




$$
\begin{gathered}
G_{s s^{\prime}, \mu, n}^{R}=-\frac{2 \mu m_{s} \gamma_{s s^{\prime}} f_{M s}}{B T_{s}}\left(w_{\|} \frac{\partial^{2} H_{s^{\prime}, n}}{\partial w_{\|} \partial \mu}\right. \\
\left.+2 \mu \frac{\partial^{2} H_{s^{\prime}, n}}{\partial \mu^{2}}+\frac{\partial H_{s^{\prime}, n}}{\partial \mu}+\frac{T_{s}}{m_{s^{\prime}}} \frac{\partial L_{s^{\prime}, n}}{\partial \mu}\right)
\end{gathered}
$$

and

$$
\begin{array}{r}
\Delta_{s s^{\prime}, n}^{R}=-\frac{n^{2}}{z_{s}^{2}} \frac{m_{s} \gamma_{s s^{\prime}} f_{M s}}{\mu B T_{s}}\left(w_{\|} \frac{\partial H_{s^{\prime}, n}}{\partial w_{\|}}\right. \\
\left.+2 \mu \frac{\partial H_{s^{\prime}, n}}{\partial \mu}-H_{s^{\prime}, n}+\frac{T_{s}}{m_{s^{\prime}}} L_{s^{\prime}, n}\right)
\end{array}
$$

Using $S(\mathbf{x})=S\left(\mathbf{X}+\boldsymbol{\rho}_{s}\right) \simeq S(\mathbf{X})+\mathbf{k}_{\perp} \cdot \boldsymbol{\rho}_{s}$ and (B6), we find

$$
\exp (i S(\mathbf{x}))=\sum_{n=-\infty}^{\infty} J_{n}\left(z_{s}\right) \exp \left(-i n\left(\varphi+\varphi_{\mathbf{k}}\right)+i S(\mathbf{X})\right)
$$

giving that the Fourier transform of the gyroaverage of $\nabla_{w} \cdot \overline{\boldsymbol{\Gamma}}_{s s^{\prime}}^{R}$ is

$$
\begin{aligned}
\left\langle\nabla_{w} \cdot \boldsymbol{\Gamma}_{s s^{\prime}}^{R}\right\rangle_{s} & =\sum_{n=-\infty}^{\infty} J_{n}\left(z_{s}\right)\left(\frac{\partial G_{s s^{\prime}, w_{\|}, n}^{R}}{\partial w_{\|}}\right. \\
+ & \left.\frac{\partial G_{s s^{\prime}, \mu, n}^{R}}{\partial \mu}-\frac{k_{\perp}^{2} \mu B}{\Omega_{s}^{2}} \Delta_{s s^{\prime}, n}^{R}\right) .
\end{aligned}
$$

This equation combined with $\mathrm{C} 3$ and $\mathrm{C} 4$ gives that the Fourier transform of the collision operator is

$$
\begin{aligned}
\left\langle C_{s s^{\prime}}^{(\ell)}\right\rangle_{s}=\frac{\partial}{\partial w_{\|}} & \left(\Gamma_{s s^{\prime}, w_{\|}}^{\lambda}+\Gamma_{s s^{\prime}, w_{\|}}^{\varepsilon}+\Gamma_{s s^{\prime}, w_{\|}}^{R}\right) \\
& +\frac{\partial}{\partial \mu}\left(\Gamma_{s s^{\prime}, \mu}^{\lambda}+\Gamma_{s s^{\prime}, \mu}^{\varepsilon}+\Gamma_{s s^{\prime}, \mu}^{R}\right) \\
& -\frac{k_{\perp}^{2} \mu B}{\Omega_{s}^{2}}\left(D_{s s^{\prime}}^{\lambda}+D_{s s^{\prime}}^{\varepsilon}+D_{s s^{\prime}}^{R}\right),
\end{aligned}
$$

where

$$
\begin{aligned}
\Gamma_{s s^{\prime}, w_{\|}}^{R} & =\sum_{n=-\infty}^{\infty} J_{n}\left(z_{s}\right) G_{s s^{\prime}, w_{\|}, n}^{R} \\
\Gamma_{s s^{\prime}, \mu}^{R} & =\sum_{n=-\infty}^{\infty} J_{n}\left(z_{s}\right) G_{s s^{\prime}, \mu, n}^{R}
\end{aligned}
$$

and

$$
\begin{array}{r}
D_{s s^{\prime}}^{R}=\sum_{n=-\infty}^{\infty}\left[\frac{J_{n-1}\left(z_{s}\right)-J_{n+1}\left(z_{s}\right)}{2 \mu z_{s}} G_{s s^{\prime}, \mu, n}^{R}\right. \\
\left.+J_{n}\left(z_{s}\right) \Delta_{s s^{\prime}, w_{\mid l}, n}^{R}\right] .
\end{array}
$$

To obtain the final result in (C20), we integrated the first two terms in $\mathrm{C} 19$ by parts in $w_{\|}$and $\mu$, and we used $\partial J_{n}\left(z_{s}\right) / \partial w_{\|}=0$ and $\partial J_{n}\left(z_{s}\right) / \partial \mu=$ $\left(k_{\perp} \sqrt{B} / \Omega_{s} \sqrt{2 \mu}\right)\left(d J_{n} / d z_{s}\right)$, where $d J_{n} / d z_{s}=\left[J_{n-1}\left(z_{s}\right)-\right.$ $\left.J_{n+1}\left(z_{s}\right)\right] / 2$.

\section{Appendix D: Derivation of the local $\delta f$ equations for the electromagnetic fields}

In this Appendix we show how to obtain (28)-(30) from (14)-(16). We only need to Fourier transform (14)-(16). In equation (14), the integral $\int d w_{\|} d \mu d \varphi \bar{h}_{s}$ can be written as

$$
\sum_{k_{\psi}, k_{\alpha}} \int d w_{\|} d \mu h_{s} \int d \varphi \exp (i S(\mathbf{X}))
$$

where $S(\mathbf{X})=k_{\psi} \psi(\mathbf{X})+k_{\alpha} \alpha(\mathbf{X})$. Using $S(\mathbf{X})=$ $S\left(\mathbf{x}-\boldsymbol{\rho}_{s}\right) \simeq S(\mathbf{x})-\mathbf{k}_{\perp} \cdot \boldsymbol{\rho}_{s}$ and (B3), we find $\int d \varphi \exp (i S(\mathbf{X}))=2 \pi \exp (i S(\mathbf{x})) J_{0}\left(z_{s}\right)$, and 28 immediately follows. Equations (29) and (30) are obtained from (15) and $(16)$ in a similar way. In equation 16. we find the integral $\int d \varphi \mathbf{w}_{\perp} \exp (i S(\mathbf{X}))$. Using $S(\mathbf{X}) \simeq S(\mathbf{x})-i \mathbf{k}_{\perp} \cdot \boldsymbol{\rho}_{s}$ and (B4), we obtain (30).

\section{Appendix E: Derivation of the local $\delta f$ flux of toroidal angular momentum}

In this Appendix we show how to obtain $46-(56)$ from (40-45. We only show in detail how to obtain (46) from the term proportional to $-(c / B) \nabla \bar{\phi} \times \hat{\mathbf{b}}$ in 40 . We then briefly cover the other terms. Writing the flux surface average explicitly, we obtain

$$
\begin{array}{r}
\pi_{s, \|}^{\phi}=\frac{m_{s} c I}{V^{\prime} \Delta \psi} \sum_{k_{\psi}, k_{\alpha}, k_{\psi}^{\prime}, k_{\alpha}^{\prime}} \int d \psi d \theta d \zeta \frac{\mathcal{J}}{B} \\
\times i k_{\alpha} \phi\left(k_{\psi}, k_{\alpha}\right) \int d^{3} w h_{s}\left(k_{\psi}^{\prime}, k_{\alpha}^{\prime}\right) w_{\|} \\
\quad \times \exp \left(i \sigma\left(\mathbf{x}, \mathbf{X}, k_{\psi}, k_{\alpha}, k_{\psi}^{\prime}, k_{\alpha}^{\prime}\right)\right),
\end{array}
$$

where we employ the abbreviated notation $\sigma\left(\mathbf{x}, \mathbf{X}, k_{\psi}, k_{\alpha}, k_{\psi}^{\prime}, k_{\alpha}^{\prime}\right)=k_{\psi} \psi(\mathbf{x})+k_{\alpha} \alpha(\mathbf{x})+k_{\psi}^{\prime} \psi(\mathbf{X})+$ $k_{\alpha}^{\prime} \alpha(\mathbf{X})$. Using $\mathbf{X}=\mathbf{x}-\boldsymbol{\rho}_{s}, \sigma$ can be simplified to $\sigma \simeq\left(k_{\psi}+k_{\psi}^{\prime}\right) \psi(\mathbf{x})+\left(k_{\alpha}+k_{\alpha}^{\prime}\right) \alpha(\mathbf{x})-i \mathbf{k}_{\perp}^{\prime} \cdot \boldsymbol{\rho}_{s}$, with $\mathbf{k}_{\perp}^{\prime}=k_{\psi}^{\prime} \nabla \psi+k_{\alpha}^{\prime} \nabla \alpha$. Using $\alpha=\zeta-q \vartheta-\Omega_{\zeta} t$, and noting that the integral over $\psi$ and $\zeta$ is over distances much longer than the perpendicular correlation length, we find that only the contributions with $k_{\psi}^{\prime}=-k_{\psi}$ and $k_{\alpha}^{\prime}=-k_{\alpha}$ do not vanish, leading to

$$
\begin{gathered}
\pi_{s, \|}^{\phi}=\frac{2 \pi i m_{s} c I}{V^{\prime}} \sum_{k_{\psi}, k_{\alpha}} k_{\alpha} \int d \theta \frac{\mathcal{J}}{B} \phi\left(k_{\psi}, k_{\alpha}\right) \\
\quad \times \int d^{3} w h_{s}\left(-k_{\psi},-k_{\alpha}\right) w_{\|} \exp \left(i \mathbf{k}_{\perp} \cdot \boldsymbol{\rho}_{s}\right) .
\end{gathered}
$$

To obtain the final result in 46, we use $d^{3} w=$ $B d w_{\|} d \mu d \varphi$ and (B3).

Equations (47)-56 are obtained in a similar way. To obtain the final expressions, we only need (B3), (B4) and

$$
\begin{array}{r}
\int d \varphi \mathbf{w}_{\perp}(\mathbf{w} \times \hat{\mathbf{b}}) \exp \left(i \mathbf{k}_{\perp} \cdot \boldsymbol{\rho}_{s}\right) \\
=2 \pi \mu B\left[\frac{2 J_{1}\left(z_{s}\right)}{z_{s}} \hat{\mathbf{b}} \times \stackrel{\leftrightarrow}{\mathbf{I}}-\frac{\mu B}{2 \Omega_{s}^{2}} G\left(z_{s}\right)\left(\hat{\mathbf{b}} \times \mathbf{k}_{\perp}\right) \mathbf{k}_{\perp}\right],
\end{array}
$$


where $G\left(z_{s}\right)=\left[8 J_{1}\left(z_{s}\right)+4 z_{s} J_{2}\left(z_{s}\right)-4 z_{s} J_{0}\left(z_{s}\right)\right] / z_{s}^{3}$. This result is obtained by realizing that $\mathbf{w}_{\perp}(\mathbf{w} \times \hat{\mathbf{b}}) \exp \left(i \mathbf{k}_{\perp}\right.$. $\left.\boldsymbol{\rho}_{s}\right)=-\Omega_{s}^{2} \hat{\mathbf{b}} \times \nabla_{\mathbf{k}_{\perp}} \nabla_{\mathbf{k}_{\perp}} \exp \left(i \mathbf{k}_{\perp} \cdot \boldsymbol{\rho}_{s}\right)$, giving $\int d \varphi \mathbf{w}_{\perp}(\mathbf{w} \times$ $\hat{\mathbf{b}}) \exp \left(i \mathbf{k}_{\perp} \cdot \boldsymbol{\rho}_{s}\right)=-2 \pi \Omega_{s}^{2} \hat{\mathbf{b}} \times \nabla_{\mathbf{k}_{\perp}} \nabla_{\mathbf{k}_{\perp}} J_{0}\left(z_{s}\right)$.

\section{Appendix F: Up-down reflection of the equilibrium}

In this Appendix we show that it is possible to find a poloidal angle definition $\theta$ for the up-down reflection of an equilibrium such that Table $\mathbb{1}$ is satisfied.

We first calculate the geometrical coefficients $B, \mathbf{B} \cdot \nabla \theta$, $\mathcal{J},|\nabla \psi|^{2}, \nabla \psi \cdot \nabla \theta$ and $|\nabla \theta|^{2}$ as functions of $\psi$ and $\theta$ for some choice of poloidal angle definition $\theta$. We invert the functions $\psi(R, Z)$ and $\theta(R, Z)$, where $R$ and $Z$ are the typical radial and axial coordinates satisfying $\nabla R \times$ $\nabla Z=\hat{\boldsymbol{\zeta}}$. We then write all the coefficients using the functions $R(\psi, \theta)$ and $Z(\psi, \theta)$. The poloidal component of the magnetic field $\mathbf{B} \cdot \nabla \theta=(\nabla \zeta \times \nabla \psi) \cdot \nabla \theta$ is written in terms of $R$ and $Z$ as

$$
\mathbf{B} \cdot \nabla \theta=\left[R\left(\frac{\partial R}{\partial \psi} \frac{\partial Z}{\partial \theta}-\frac{\partial Z}{\partial \psi} \frac{\partial R}{\partial \theta}\right)\right]^{-1} .
$$

The Jacobian $\mathcal{J}=|\mathbf{B} \cdot \nabla \theta|^{-1}$. The products $|\nabla \psi|^{2}$, $\nabla \psi \cdot \nabla \theta$ and $|\nabla \theta|^{2}$ are

$$
\begin{gathered}
|\nabla \psi|^{2}=\left(\frac{R}{\mathcal{J}}\right)^{2}\left[\left(\frac{\partial R}{\partial \theta}\right)^{2}+\left(\frac{\partial Z}{\partial \theta}\right)^{2}\right], \\
\nabla \psi \cdot \nabla \theta=-\left(\frac{R}{\mathcal{J}}\right)^{2}\left(\frac{\partial R}{\partial \psi} \frac{\partial R}{\partial \theta}+\frac{\partial Z}{\partial \psi} \frac{\partial Z}{\partial \theta}\right)
\end{gathered}
$$

and

$$
|\nabla \theta|^{2}=\left(\frac{R}{\mathcal{J}}\right)^{2}\left[\left(\frac{\partial R}{\partial \psi}\right)^{2}+\left(\frac{\partial Z}{\partial \psi}\right)^{2}\right]
$$

The magnitude of the magnetic field is $B^{2}=\left(I^{2}+\right.$ $\left.|\nabla \psi|^{2}\right) / R^{2}$.

For an equilibrium given by $R(\psi, \theta)$ and $Z(\psi, \theta)$, the equilibrium described by $R(\psi, \theta)$ and $-Z(\psi, \theta)$ is the updown reflection. This is a way of choosing the poloidal angle definition $\theta$ for the up-down reflection. With this choice, equivalent to replacing $Z$ by $-Z$ in $(\mathrm{F} 1)-(\mathrm{F} 4)$, we obtain most of the results in Table I. The poloidally varying quantities $n_{s}, p$ and $\partial p /\left.\partial \psi\right|_{R}$ remain the same because they depend on $\theta$ only through the function $R(\psi, \theta)$, which is unchanged upon up-down reflection.

\section{Appendix G: Proof of the results in Table III}

In this Appendix we evaluate the coefficients in Table III and express them as functions of other coefficients whose behavior under the transformations in Table II we know.

Using $\nabla \alpha \times \mathbf{B}=(\nabla \psi \cdot \nabla \alpha) \nabla \alpha-|\nabla \alpha|^{2} \nabla \psi$, we find

$(\nabla \alpha \times \mathbf{B}) \cdot \nabla \theta=(\nabla \alpha \cdot \nabla \psi)(\nabla \alpha \cdot \nabla \theta)-|\nabla \alpha|^{2}(\nabla \psi \cdot \nabla \theta)$

Using $\nabla R \times \hat{\boldsymbol{\zeta}}=-\nabla Z$, we find

$$
(\nabla R \times \hat{\boldsymbol{\zeta}}) \cdot \nabla \alpha=-\frac{\partial Z}{\partial \psi} \nabla \psi \cdot \nabla \alpha-\frac{\partial Z}{\partial \theta} \nabla \theta \cdot \nabla \alpha
$$

Finally, the coefficients $|\nabla \alpha|^{2}, \nabla \alpha \cdot \nabla \psi$ and $\nabla \alpha \cdot \nabla \theta$ are

$$
\begin{array}{r}
|\nabla \alpha|^{2}=\frac{1}{R^{2}}+\left(\vartheta \frac{\partial q}{\partial \psi}+q \frac{\partial \vartheta}{\partial \psi}+t \frac{\partial \Omega_{\zeta}}{\partial \psi}\right)^{2}|\nabla \psi|^{2} \\
+\frac{2 I}{R^{2} \mathbf{B} \cdot \nabla \theta}\left(\vartheta \frac{\partial q}{\partial \psi}+\right. \\
\left.+\frac{\partial \vartheta}{\partial \psi}+t \frac{\partial \Omega_{\zeta}}{\partial \psi}\right) \nabla \psi \cdot \nabla \theta \\
+\left(\frac{I}{R^{2} \mathbf{B} \cdot \nabla \theta}\right)^{2}|\nabla \theta|^{2}
\end{array}
$$

$$
\begin{array}{r}
\nabla \alpha \cdot \nabla \psi=-\left(\vartheta \frac{\partial q}{\partial \psi}+q \frac{\partial \vartheta}{\partial \psi}+t \frac{\partial \Omega_{\zeta}}{\partial \psi}\right)|\nabla \psi|^{2} \\
-\frac{I}{R^{2} \mathbf{B} \cdot \nabla \theta} \nabla \psi \cdot \nabla \theta
\end{array}
$$

and

$$
\begin{aligned}
\nabla \alpha \cdot \nabla \theta=-\left(\vartheta \frac{\partial q}{\partial \psi}+q \frac{\partial \vartheta}{\partial \psi}\right. & \left.+t \frac{\partial \Omega_{\zeta}}{\partial \psi}\right) \nabla \psi \cdot \nabla \theta \\
& -\frac{I}{R^{2} \mathbf{B} \cdot \nabla \theta}|\nabla \theta|^{2}
\end{aligned}
$$

with the function $\vartheta(\psi, \theta)$ defined in 18 .

With these expressions, and employing Table I, we can show that transformation I, which is an up-down reflection and a sign change in $\Omega_{\zeta}$ and $\partial \Omega_{\zeta} / \partial \psi$, transforms the coefficients as in Table III. We can prove the same for transformation II, which is an up-down reflection and a sign change in $I$.
[1] J.W. Connor, T. Fukuda, X. Garbet, C. Gormezano, V. Mukhovatov, M. Wakatani, the ITB Database Group, and the ITPA Topical Group on Transport and Internal
Barrier Physics, Nucl. Fusion 44, R1 (2004).

[2] J.W. Connor, and H.R. Wilson, Plasma Phys. Control. Fusion 42, R1 (2000). 
[3] W. Dorland, F. Jenko, M. Kotschenreuther, and B. N. Rogers, Phys. Rev. Lett. 85, 5579 (2000).

[4] J. Candy, and R. E. Waltz, J. Comput. Phys. 186, 545 (2003).

[5] T. Dannert, and F. Jenko, Phys. Plasmas 12, 072309 (2005).

[6] A.G. Peeters, Y. Camenen, F.J. Casson, W.A. Hornsby, A.P. Snodin, D. Strintzi, and G. Szepesi, Comput. Phys. Commun. 1802650 (2009).

[7] A.G. Peeters, C. Angioni, and D. Strintzi, Phys. Rev. Lett. 98, 265003 (2007).

[8] R.E. Waltz, G.M. Staebler, J. Candy, and F.L. Hinton, Phys. Plasmas 14, 122507 (2007); 16, 079902 (2009).

[9] Y. Camenen, A.G. Peeters, C. Angioni, F.J. Casson, W.A. Hornsby, A.P. Snodin, and D. Strintzi, Phys. Rev. Lett. 102, 125001 (2009); Phys. Plasmas 16, 062501 (2009).

[10] C.M. Roach, I.G. Abel, R.J. Akers, W. Arter, M. Barnes, Y. Camenen, F.J. Casson, G. Colyer, J.W. Connor, S.C. Cowley, D. Dickinson, W. Dorland, A.R. Field, W. Guttenfelder, G.W. Hammett, R.J. Hastie, E. Highcock, N.F. Loureiro, A.G. Peeters, M. Reshko, S. Saarelma, A.A. Schekochihin, M. Valovic and H.R. Wilson, Plasma Phys. Control. Fusion 51, 124020 (2009).

[11] F.J. Casson, A.G. Peeters, Y. Camenen, C. Angioni, W.A. Hornsby, A.P. Snodin, D. Strintzi, and G. Szepesi, Phys. Plasmas 16, 092303 (2009).

[12] F.J. Casson, A.G. Peeters, C. Angioni, Y. Camenen, W.A. Hornsby, A.P. Snodin, and G. Szepesi, Phys. Plasmas 17, 102305 (2010).

[13] M. Barnes, F.I. Parra, E.G. Highcock, A.A. Schekochihin, S.C. Cowley, and C.M. Roach, Phys. Rev. Lett 106, 175004 (2011).

[14] E.G. Highcock, M. Barnes, A.A. Schekochihin, F.I. Parra, C.M. Roach, and S.C. Cowley, Phys. Rev. Lett 105, 215003 (2010).

[15] A.G. Peeters, and C. Angioni, Phys. Plasmas 12, 072515 (2005).

[16] R.R. Dominguez, and G.M. Staebler, Phys. Fluids B 5, 3876 (1993).

[17] F.I. Parra, and P.J. Catto, Plasma Phys. Control. Fusion
52, 045004 (2010); 52, 059801 (2010).

[18] F.I. Parra, and I. Calvo, Plasma Phys. Control. Fusion 53, 045001 (2011).

[19] F. L. Hinton, and S. K. Wong, Phys. Fluids 28, 3082 (1985).

[20] J. W. Connor, S. C. Cowley, R. J. Hastie, and L. R. Pan, Plasma Phys. Control. Fusion 29, 919 (1987).

[21] P. J. Catto, I. B. Bernstein, and M. Tessarotto, Phys. Fluids 30, 2784 (1987).

[22] M. Artun, and W.M. Tang, Phys. Plasmas 1, 2682 (1994).

[23] H. Sugama, and W. Horton, Phys. Plasmas 5, 2560 (1998).

[24] A.G. Peeters, D. Strintzi, Y. Camenen, C. Angioni, F.J. Casson, W.A. Hornsby, and A.P. Snodin, Phys. Plasmas 16, 042310 (2009).

[25] I.G. Abel, G.G. Plunk, E. Wang, M. Barnes, S. C. Cowley, W. Dorland, and A. A. Schekochihin, Multiscale Gyrokinetics for Rotating Tokamak Plasmas I: Fluctuations, Transport and Energy Flows (in preparation).

[26] M.N. Rosenbluth, W.M. MacDonald, and D.L Judd, Phys. Rev. 107, 1 (1957).

[27] G.W. Hammett et al., Bull. Am. Phys. Soc. (2006), abstract VP1.136.

[28] A.N. Simakov, and P.J. Catto, Plasma Phys. Control. Fusion 49, 729 (2007).

[29] P.B. Snyder, and G.W. Hammett, Phys. Plasmas 8, 744 (2001).

[30] F. Jenko, and W. Dorland, Plasma Phys. Control. Fusion 43, A141 (2001).

[31] A.M. Dimits, G. Bateman, M.A. Beer, B.I. Cohen, W. Dorland, G.W. Hammett, C. Kim, J.E. Kinsey, M. Kotschenreuther, A.H. Kritz, L.L. Lao, J. Mandrekas, W.M. Nevins, S.E. Parker, A.J. Redd, D.E. Shumaker, R. Sydora, and J. Weiland, Phys. Plasmas 7, 969 (2000).

[32] G.W. Hammett, M.A. Beer, W. Dorland, S.C. Cowley, and S.A. Smith, Plasma Phys. Control. Fusion 35, 973 (1993).

[33] I. G. Abel, M. Barnes, S. C. Cowley, W. Dorland, and A. A. Schekochihin, Phys. Plasmas 15, 122509 (2008). 